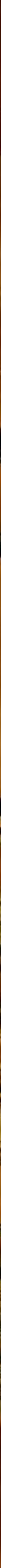




\section{HARVARD UNIVERSITY.}

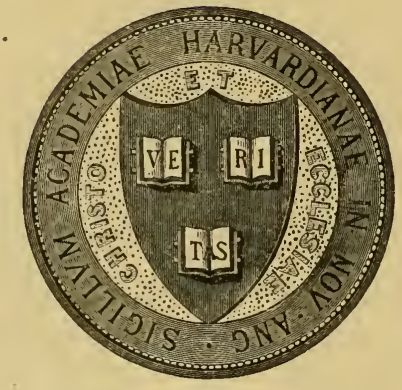

\section{LIBRARY}

OF THE

MUSEUM OF COMPARATIVE ZOÖLOGY $5,400^{2}$

GIFT OF

ftarrand college libary

April 3, 1923.

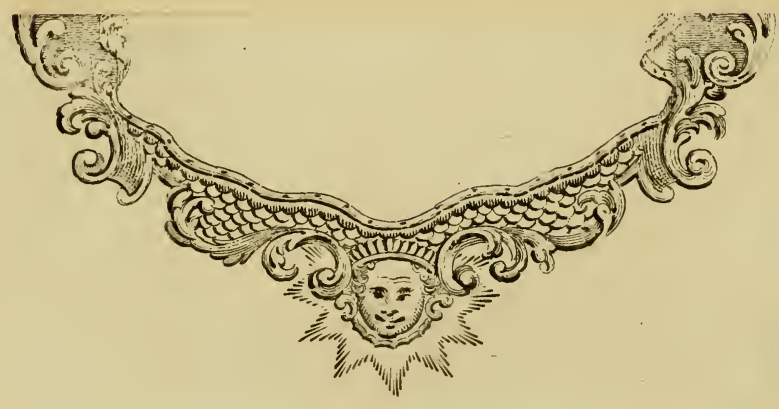


$\begin{array}{lll}\text { APR } & \mathbf{3} & 1923\end{array}$ 







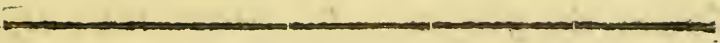

\section{N D I C E}

D' ITTIOLOGIA SICILIANA

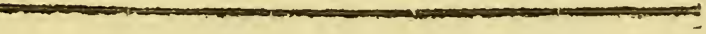


Admirons des poissons, les formes et les voyages. Tour à tour visitant nos flenves, et nos rivages, Ou hùtes sedentaires receiès dans nos caux Nous offrant on tribut lears immenses troupcaux. 


\title{
I $\mathbb{N}$

\section{D' itTílogia siciliana}

\author{
0 S S I A
}

CATAlOGO METODICO DE1 NOMI LATIN1: ITALIAN1, E SIClLIAN1 DEl PESC1, CHE SI RINVENGONO IN SICILIA

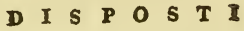

SECONDO UN METODO NATURALI

$$
\text { I S E G U I T O }
$$

DA UN APPENDICE CHE CONTIENE LA DESCRIZIONE DI ALCUNI NUOVI PESCI SICILIANI

ILLUSTRATO DA DUE PIANCE.

OPUSCOLO DEL SIGNORE

C. S. RAFINESQUE SCHMALTZ

C M E S S I N A

TRESSO GIOVANNI DEL NOBOLO

CON APPROVAZZIONE

1810 . 


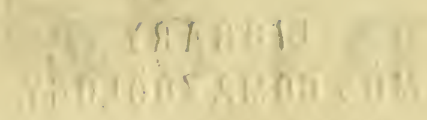

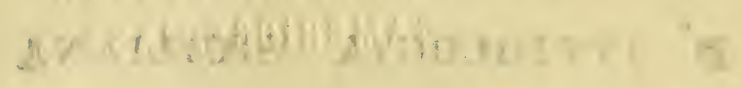

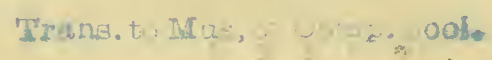

- $\begin{aligned} & \text { : } \\ & \text { and }\end{aligned}$

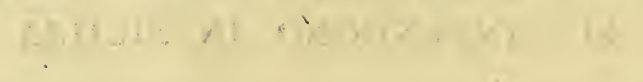

$\operatorname{tinh} 4 \operatorname{li}^{2}$

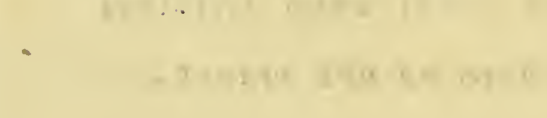




\title{
AL SIGNORE D. ANTONIO ARROSTO.
}

PREGIATISSIMOSIGNORE

\begin{abstract}
$\mathbf{M}_{\mathrm{I}}$
I permetta di dargli nna pruova della stima, che ho concepito pes. lei, coll' offrirgli questo piccolo Opuscolo, quale debole omaggio è dourto al di lei merito, e serve a mostrargli la mia gratitudine pelle notizie com municatemi sopra le piante, ed i pesci dei contorni di Messina nel mio brieve soggiorno in quella Città l' anno scorso. E sicuro che verrà grạ dirne l' attenzione, mi dichiaro
\end{abstract}

Palermo il dì 15. Maggio 1810.

Ii di lei servo 


\section{CONTENUTO DELLE DUE PIANCIE.}

Tarola 1. figura 1. Luvarus imperialis . N. 290.

fig. 2. Oxycephas Scabrus N. 42.

fig. 3. Argyctius quadrimaculatus N. 231.

Tavola 2. figura 1. Chlopsis bicolor. N. 313.

fig. 2. Oxystomus hya linus . N. 374。

fig. 3. Helmictis punctatus N. 375 .

fig. 4. Nerophis maculatus N. 271.

6.g. 5. Myctophum punctatum . N. 258.

APIEGAZIONE di ALCUNI 'SEgNI \&C.

- Significa che quella specie di Pesce, si ritrova pure nei fiumi stagni, ed aque dolci, ò vi dimora esclusivamente .

*. Significa che è un pesce descritto da me per la prima volta, ¿̀ sconosciuto da Linneo, Lacepede, ed altri autori .

11 primo nome del pesce è l' ittiologo latino, il secondo l' Ittịologo italiano, e gli altri in corsivo sono i nomi volgari Siciliani. 


\section{( $\gamma$ ) \\ BREYE INTRODUZIONE.}

1

RA i numerosi rami della Zoologia, essendomi particolarmente dato a studiare quello che tratta dei Pesci, ed avendone osser. vati in Sicilia nel corso di cinque anni una infinita varietà, aveva concepito l' idea di pubblicarne la compita Storia naturale in La. tino, o Francese, credendo essere alfine giunto a conoscere quasi tutti quei , che vivono nelle aque di questa 1sola : non di meno pensando che di quando in quando me se $\mathrm{n}^{\prime}$ offrono dei nuovi, $\mathrm{c}$ che sia molto probabile che molti abbiano tuttora sfugite le mie ricerche, hò creduto opportuno di differirne per qualche tempo la pub. blicazione, e di stampare frattanto anticipatamente questo Catalogo metodico di quei, che hò già rinvenuti, con i loro nomi ittiologici latini, ed italiani, ed i volgari Siciliani, quali sono per lo più quei usitati in Palermo luogo del mio soggiorno, e dove furono fatte la maggiore parte delle mie osservazioni, e ricerche, affine di dare ai naturalisti una idea della ricchezza di questo ramo d' 1storia Naturale in questa parte del Mediterraneo, dove credo che il numero delle specie di Pesci supera quello di qualunque altra parte del globo di simile estenzione, ed anche per pregare qualunque osservatore siciliano di comunicarmi le sue scoperte, per arricchirne in seguito la mia sopracitata opera; bramerei particolarmente dei rischiaramenti sopra la Sínonimia, c la corríspondenza dei nomi diversi, che si danno ai Pesci, nelle diverse parti di questo regno, a quale oggetto aggiungo alla fine di questo Indice un Catalogo di alcuni nomi, che si dauno a Catania, a Messina \&c. a certi pesci, che non avendo osservati nei luoghi dove sono così nominatí, non ho potuto ra gguagliare alle specie da me mentovate: Qualunque altra osservazione, elucidazione, ó giusta critica saranno da me ricevate cou sommo piacere, e grata riconoscenza . 


\section{$(8)$}

Hò classificati i Pesci di Sicilia secondo un nnovo metodo baturale, frutto di dieci anni di studj, ed osservazioni in America, ed Europa, e per elucidare il quale mi sono pure servito delle opere di Lacepede, Cusier etc.

Ogni Pesce che descrisse Linneo ò il suo continuatore Gmelin nell' ultima edizione del Sistema Naturce viene seguito in questo Indice dalle Lettere Linn., quelli segnati Lac. furono ilescritti dopo loro da Lacepede nella sua Storia Naturale dei Pesci, e hò marcati $\boldsymbol{R} a f$. quelli di cui hò dovuto cambiare il nome, $\boldsymbol{R} a f$. Car. quelli che io hò fatto conoscere il primo nel mio Opuscolo intitolato, Caratteri di alcuni nuovi generi, e nuove specie d' animali, e Piante della Sicilia, e finalmente Raf. app. quelli che hè osservati dopo che composi 'quell' opera, e di cui darò un breve raguaglio nell' appendice in fine di questo indice, affinchè ogni lettore avendo riferenza alle opere di Linneo, Lacepede, e le mie, possa ottenere a suo piacere la eognizione dei pesci, che avrò enumerati, e due rami che contengono le figure di otto Pesci di generi, e specie nuove, serviranno a maggiormente illustrare il prea sente opuscolo, 


\section{( 9) \\ $\mathbb{N} \mathbb{D} \mathbb{I} \mathbb{C}$}

\section{D' I'T'TIOLOGIA SICILIANA.}

\section{PRIMA SOTTO-CLASSE - 1 POMNIODI.}

Branche compite, e proviste di un opercolo, e di una mem Brana branchiale.

\section{PRIMA DIVISIONE. I GIUGULARI.}

Ale inferiori situate sotto la gola, ò anteriormente all' ale pettorali.

\section{PRIMA SEZIONE. I CORISOSTALMI.}

Un occhio da ogni lato del capo.

\section{ORDINE. I BLENNID1.}

Corpo conico v̀ lanceolato, ale giugulari con un solo raggio, con più d' uno riuniti assieme senza membrana intermedia, oper。 colo mutico .

1. Blennius tentacularis. Linn. Blennio tentacolare. Bausa ucchiuta.

2. gattorugine. Linn. Blennio gattorugine. Pakus" nu imperiali.

3. - barbatus . Raf. (Blennius mediterraneus, Lac. Gadus mediteraneus . Lin.) Blennio barbato . Mustia imperiale.

A. - galerita. Linn. Blennio galerita. Pesce lonara ò Bavusa cu tupè .

* 5. mustea. Raf. car. sp. 65. Blennio mustea. Mastca, ò mustia. 
* 6. Blennius lupus. Raf. car. sp. 66.

Blennio lupe.

Pesce

$$
\text { lири . }
$$

$* 7$. labrus . Raf. car. sp. 67. - do bavusone.

* 8 . Blennius patuanus. Raf. car. sp. 74 . Blenni patuvano. Patuoanu, ò Pataune.

* 9. gonocephalus . Raf. app. sp. 1. Blennio gonoec。 falo. Bavusa gurgiune .

t*10.

Bavusa immuruta nel val di mazzara, $B a$ s buccia nel val demone, e Tombarelli nel val di noto.

* 11. Aluviatilis. Raf. car. sp. 78. Blennio di fiume. Bavusa de sciume, ò minusa.

12. nebulosus. Raf. car. sp. 76. Blennio nuvolose. Lonora di nicori.

13. - pholis. Linn. Blennio folide. Bavusa impiriali.

+14 . gobioides . Raf. app. sp. 2. Blennio ghiozzo。 Minusa ò Bavusedda, ò Gurgiuneddu. 7 15. Variegatus. Raf. carr. sp. 75. Blennio variegate. Tordo lonora.

* 16. Vividus. Raf. car. sp. 70. Blennio Vivace. Bavusa.

17. Variabilis. Raf. car. sp. 71. Blennio varias bile. Spirdottu, Sperdoto, ̀̀ Spirda. * 18. Spirdottus . Raf. ear. sp. '72. Blennio spirdoto come il precedente. $\Rightarrow$ 19. Violaceus. Raf. (Blennius fasciatus. Raf. car. 5P 


\section{(11)}

73.) Blennio violaceo. come $i$ due precedenti.

20. Blennius Julioides . Raf. car. sp. 69:I ? Blennio julino.

Viola bavusa.

21. Longus . Raf. car. sp. 68. Blennio lungo . bausedda.

* 22. Physophthalmús . Raf. car. sp. 64. Blennio oc. chi-gonfi Mustia impiriali.

23. Phycis (Raf. car. gen. 26.) ocellaris . Rat. ( Blennius occellaris . Lin: Blennius lepus, Lac. ). Fice oculato .Patuvanu ò pesce lebre.

24. - Albidus . Raf. (Gadus albidus, Linn. Blennius gadoides . Lac.) Fice bianco. Stoca. pesce impiriali.

25. - Lepus. Raf. (Blennius phycis. Linn. Lac. ) . Fi. ce lepre. Pesce lebre, ò Bavusuni.

26. Macrophthalmus. Raf. car. sp. 62. Fice grossoc. chio. Stocapesce, Stocafissu, ò Gruncu impiriali .

27. Gaidropsarus inustellaris. Raf. app. gen.'1. sp. 3. Ghe. dropsaro mustellaro. Mustidda impiriali.

\section{ORDINE. 1 GAD1N1.}

Corpo conico, ò lanceolato, ale giugulari con diversi raggi riuniti da meinbrane, opercolo mutico membranoso, occhi distan¿, e laterali".

28. Gadus minutus, Linn. Gado minore. Pesce ficu. Mancanu, ò Bacaficu nel ral di Noto, Muncana a Malia. 
29. Gadus Blennivides Linn. Gado blennio: Mcrluzzu impiriali.

30. Onus riali . ( Raf. Merluccius, Raf. car. gen. 25.) Mer. luccius. Raf. ( Garlus merluccius. Linn. ) Nasello. merluzzo. Merluгzo, Merluzzu, ¿ Mirruะzu:

- 31. - Smiridus Raf. (Merluccius Smiridus . Raf. car. sp. 61. ) Nasello Smirido. Smiriddu,

a Messina Smidiru.

32. Mustellus. Raf. (Gadus mustella . Linn. Phycis punctatus. Raf. car. sp. 63.) Nasello Mustello. - Mustedda, Mustidde, is pesce Lupu.

- 33. Strinsia tinea . Raf. app. genn. 2. sp. 4. Strinsia tinea . Pesce moddu, ò Tenca di mare.

\section{ORDINE. 1 TRACHFNIDI.}

Corpo conico, d̀ lanceolato, ale giugulari con diversi raggi riuniti da membrane, opercolo dentato, ciliato, ò spinoso, acchj arvicinati, e situati sopra il capo :

31. Callionymus lyra. Línn. Callionimo rondínella .

Vellisu, ò pesce anpiscica impiriali.

35. Dracunculus . Linn.

Callionimo dragoncello. come il precedente.

96. -_._. Maculatus Raf. car. sp. 60. Callionimo macchiate. conce $i$ precedcriti,

37. Uranoscopus Scaber. Línn. Uranoscopo ruvide.

Coce ciu impiriali a Catania Coccia, ò Cozzolo. 
* 38. Uranoscopus Cocius. Raf. car. sp. $59 . \quad$ Uranoscopo concio Coccio, Cocciu, ò Gocciu.

39. Trachinus vividus Lac. ( Tr. draco. Linn.) Trachino ragana. Tracena, ò Tragina, Trachio a Catania .

* 40. - Vainus. Raf. car. sp. 57. Trachina Vaina, Vaina; ò Tracena d'Arca.

- 41. Corystion, Striatus. Raf. ( C. mustazola . Raf. car. gen。 24. sp. 58. ) Coristio rigato. Mu stazola a Palermo. Cocama? a Catania. Matarello nel val di Noto.

* 42. Oxycephas Scabrus Raf. car. gen. 27. sp. 79. Ossice。 falo ruvido. Pizsune . Tav, 1. fig. 2。

\section{V. ORDINE. 1 CURTSI .}

Corpo ovale, $c$ compresso, ate giugulari con i raggi rianiti dan membrane, opcrcolo mutico, occhj distanti, e laterali . 43. Chrysostroma Fiatoloides. Lac. Crisostroma fiatoloide. Fiatulic impiriali.

SECONDA SEZIONE.

1 PLEUROSTAMI.

I dice occhj' dell' istesso lato del capo . V. ORDINE. GLI AGHIRINI . Senza ale pettorali.

* 44. Symphurus nigrescens.. Raf. app. gen. 3. sp. 5. Sine foro nerastro. Linguatidda. 


\section{(14) \\ V1. ORDINE. 1 PLERONETTI.}

45 Solea (Raf. app. gen. 4.) buglossa. Raf. ( Pleuronectes solea Linn.) Sogliola comune . Linguata.

a Messina Palaja. a Catania Linguatu.

46. Limanda. Raf. ( Pleuronectes Linguata Linn.)

Sogliola limanda. Lema, ò Lima, Passari .

47. Platessa . Raf. (PI. platessa Linn.) Sogliola pianosa -Pianussu, ò Passera.

48. Rhomboide. Raf. app. sp. 6. ( PI. limanda . var. Lac. ) Sogliola romboide. Rumbu impi-. riali .

49. Cithara. Raf. app. sp. 7. Sogliola citara . Cantinu .

50. - pegusa . Raf. ( Pl. pegusa . Lac.) Sogliola pegusa . Linguata ucchiuta.

* 51. - Arnoglossa. Raf. app. sp. 8. Sogliola arnaglossa. Linguata liscia.

* 52.-cynoglossa. Raf. app. sp. 9. Sogliola linguacane - Linguala mavista.

53. Scophthalmus ( Raf. app. gen. 5. ) maximus. (Pleuronectes maximus Linn.) Rombo massimo. K’umolo impiriali.

54. - Rhombus. Raf. (Pl. rhombus Linn.) Rombo comune. Rumbu, ò Linguata masculu. a Messina Passera.

55. - diurus . Raf. app. sp. 10. Rombo doppiacoda. Rambu dupiacudes 


\section{( 15$)$}

* 56. Bothus rumolo. Raf. car. gen. Q3. sp. 5 .

Boto rumolo. Rumolo. a Catania Lumeru.

* 57. Tappa. Raf. car. sp. 55. Boto tappa .

Tappa. a Catania Panta

* 58. - Imperialis . Raf. car. sp. $56 . \quad$ Boto im. periale . Tappa impiriali, ò Linguata im. piriali.

\section{SECONDA DIVISIONE。 I TORACICI .}

Ale inferiori situate sotto it petto, ò al disotto dell' Ale pettorali.

\section{PRIMA SEZIONE - GLI EMISFERONOTI.}

Corpo molto compresso, corto, ovale, ò quasi romboidale, alto quanto è lungo, ò piu alto, che lungo, e col dorso æzolto curvo.

\section{V11. ORDINE . I SEJENIDI . \\ V11I. ORDINE。 I ZEUS1D1。}

Una membrana verticale, e trasversale sotto il labro infe. xiore, opercolo mutico, senza raggi, ò spine isolate sopra il dor• so, senza denti mobili, e flessibili, e senza ale squamose. 59. Zeus faber. Linn. Zeusi fabro Pesce ga'lo o pesce gaddu nel val di Mazzara. Pesce palı, - Gaddu Marinu nel val demone. Pesce Sanpieri nel val di noto, c Larnata a Malta .

67. Capros aper. Lac. ( Zeus aper. Linn.) Capro arrizzato. Pesce Tariolo. 1X. 
IX. ORDINE.

X. ORDINE.

X1. ORDINE.

X11. ORDINE.

SECONDA SEZ1ONE.
GL1 EQUE DIN1。

1 CHETODONIDI :

GL1 ACANTURIN1.

GLI OLACANTINI.

1 TOSSONOTI .

Corpo allungato, ovale ellittico, o fusiforme, e piu, ล sueno compresso, piir lungo, che alto, e con il dorso più, o meno curvo.

\section{X111. ORDINE. I PERCIDI .}

Opercolo dentillato, ò spinoso.

- 61. Lepipterus fetola . Raf. Lcpterus fetula . Raf. car. sic. gen. 33. sp. 142.) Lepitero fetula. Fetula a Catania Fetolo.

62 Perca umbra . Lac. ( Sciena cirrosa Linn.) Perca om. brina. Umbrina impiriali, ò Budagìa, ò Umbra. $+63$. Fluviatilis . Linn. Perca fluviatile. Percies di Sciumi.

61. Brunichi . Lac. (P. pusilla. Linn.) Perca piccola. Pesce serra.

5j. Diacantha Lac- Perca duespina. Serrania; o Serraina a Palermo, e nel val di Mazzara Bodasce, Bodagie, ò Boragie nel val demoa ne, e nel val di noto.

66. Punetata Lac. non Linn. Perca puntata. come la precedente. 


\section{$(17 \cdot)$}

+ 67. Sciena Umbra Linn. Ombrina comune. Umbrina.

68. Cappa. Linn.

Ombrina cappa.

Scrrania di niuri.

69. Unimaculata. Linn. Ombrina macchiata . Mineluz $\approx$.

70. Lopharis mediterraneus. Raf. car. gen. 3\%. (Centropnmus lophar. Lac. Perca lophar. Linn.) Lofare mediterraneo. Percia di gramigna..

+ 71. Centropomus lupus Lac. (Perca punctata Linn.) Centropomo lupo. Spinula a Palermo, e nel val di Mazzara. Spinota nei valli Demone, e Noto.

72 Lineatus . Lac. Centropomolineato ... Spime la lineata, ò Impiriali.

73. Holocentrus Sogo . Lac. Olocentro Sogo - Scrranec di fango nel val Mazzara . Bodagia nei valli Demone, e Noto.

74.-Chanus Raf. (II. chani . Lac., Labrus chanus Linn.)

Serrania, ̀̀ Bodagia di solo.

75. - Cernus. Raf. (Hol. post. Lac., Perca cernua. Linn.) Olocentro cerno. Cernia. a Malta Cerna. 76. Marinus Lac. (Perca marina Linn.) Olocentro marino . Serrania di niuri .

77._ Gigas. Raf. ( Hol. merou. Lac., Perca gigas. Linn. Olocentro gigante . Cernia.

78. Aylopon anthias. Raf. car. gen. 36. (Anthias barbier, Bloch . Lutianus anthias. Lac. Labrus anthias. Linn.) $\underset{C}{\text { Elopone antia. Monacedda diforle. }}$ 
69. Lutianus mediterrancus . Lac. (Perca mediterranea . Lina.3) Lutiano mediterranco. Percia a Palermo. Boragia a Messina, e Catania.

80. Adriaticus . Lac. ( Labrus adriaticus . Linn.) Lutiano adriatico. Perciuda a Palermo.

81. Massiliense . Lac. (Labrus Unimaculatus . Linn.) Lutiano marsigliese. Percia di solo.

82. Brunichi . Lac. ( Labius fuscus Linn.) Lutia no fosco. Percia di Niuri.

8::- Olivaceus . Lac. (Labrus olivaceus . Linn.)

Lutiano ol ivaceo. Pesce Sapuni.

84. - Serran. Lac. (Perca cabrila Linn.) Lutiane

Serrano. Serranict, ò Seraina nel val di

Mazzara . Bodagie, Bodage, ò Burrace nel val demone, e nel val di Noto.

$* 85$. Crapa . Raf. car. Sic. sp. 141. Lutiano capret. to. Crapa, ò Pesce Crapa.

\section{ORDINE. GL1 SCARID1.}

Mascelle ossee in vece di denti, opercolo mutico.

86. Scarus cretensis. Raf. (Scarus Kakatoe Lac. Labrus crea tcasis. Linn.) Scaro cretico. Pesce $\mathbf{P a}$. paģaddu 。

\section{ORD1NE。 GL1 ACANT1 .}

Dei raggi, spine, ò appendici isolati sopra il dorso, ò die. to l'ano, Opercolo matico, mascelle non ossee.

57. Centronotus glaucus Lac. Centronoto glauco. Cer* viola impiriali. 


\section{( 19 i}

* 88. Centronotus Binotatus. Raf. car. síc. sp.119. Centronoto Macchiato . Ciodana, o Cionera, a Palermo. Itala a Messina .

89. Hypacanthus Vadigo. Raf. car. gen. S2. (Centronotus Vadigo, Lac.) 1pacanto vadigo.

Cervioia.

- 90. Naucrates fanfarus. Raf. car. gen. 33. sp. 120. Naucrate fanfaro. Fanfaro, Infanfaro, ò pesce fanfaru. nel val di Mazzara, Pampana a Messina .

- 91. Centracanthus cirrus. Raf. car. gen. 31. sp. 118. Centracanto cirro. Cirru a Palermo, Pesce di Umbra a Catania .

- 92. Notognidion Scirenga . Raf. car. sic. gen. 31. sp. 124. Notognidio Scirenga. Scirenga Impirialio 7. 99. Gasterosteus pungitius. Linn. Spinarola pungente . Spinarolu.

\section{XV1. ORDINE. GL1 SCOMBERIN1 .}

Divesse piccole ale al disopra, ed al disotto della coda, opercolo mutico, mascelle non ossee, e senza raggi, Spine, ò appeadici sopra il derso, ò dietro l' ano .

94. Scomber thymnus. Linn. Sgombro tonno. Tunne 。

95. _- Alalunga . Linn. Sgombro alalunga . Ala. longa, a Malta Accola.

96. Scombrus . Linn. Sgombro comune. Scur mu , ̀̀ Scombru in Palermo. Strumbu a Meșsina. Scrumiu a Catania. Sganbirri a Siracusa, e nel val di Now. $\quad 27$. 


\section{( 20$)$}

97. Scomber Colias. Linn. Sgombro laceria

Scurmu Ins piriali, a Messina Scarmi?

$-98$. Macrophithalmus . Raf. app. sp. 11. Sgombro grand' occhi. Scurmu grandocohiz.

99. - Pelamis. Linn. Sgombro bonito. Palamitu Impiriali, a Messina? Pslamatu.

* 100. Palamitus . Raf. car. sp. 121 . Sgombro pala. mito . Palamitu. a Catania Pisantuni .

* 101. Bisus . Raf. car. sp. 122. Sgombro biso . Bisu, Mpisu, c Tunnachiu nel val di Mazzara . Appicatu nel val demone. Sgamiru a Catania, e nel val di Noto.

*10\%. Aletteratus . Kaf. car. sp. 123.

Sgombro alette. rato . Aletteratu, ò Litteratu nel val di Maz. zara. Covcuritu a Messina, Catania, e Siracusa . 103. Sarda . Lac. Sggombro Sardo . Covarilu Im. piriali.

\section{XVI1. ORD1NE G GLI SPARIDI.}

Opercolo mutico, Mascelle non ossec, senza raggi, spine, se appondici sopra il dorso, ne dietro l' ano, e senza piccole ale sopra, e sotto la coda.

101. Trachurus saurus Raf. (Caraux trachurus Lac. Scomber tracĥ̀urus Linn.) Trachuro Sauro . Sauru. * 10ว. Impirialis Raf. car. gen. 30. sp. 116. Trachuro impcriale. Scuuru impiriali a Catania Sauru reali .

- 106. Aguilus . Raf. car. sp. 115. Trachuro aguilo . Aguilu, Aquila, ò Laguja nel val di Mazza. ra. 
ra. Aghila, ò Laquia nel val demone, ect Aloga nel val di Noto.

* 107. Tracorus. Aticciolus Raf. car. sp. 117. Trachuro aliciola . Alieciola, Ariciola, Licciuola, ò Liciolu.

* 108. Fasciatus . Raf. app. sp. 12. Trachuro fasciato . Liciola, ò Ariciola impiriali .

* 109. Lepodus Saragus . Raf. car. gen. 40. sp. 14t. Lepodo Sarago: Saragu impririali. ò Scarzt impi-. riali a Catania Pesee Luna.

110. Cheilinus Scarus Lac. (Labrus scarus Linn.) Chelina Scaro. Scaru, ò Scauru.

* 1I1. Symphodus fulvescens . Raf. car. gen. 29. sp. 114. Sin• forlo fulvo . Russaliddu, ò Trumbetta .

112. Labrus hepatus Linn. Labro epato. Lappanu Saragul 113. Cappa Linn. Labro eappa . Lappam . 114. Pavo. Linn. Labro pavone. Lappanu beddu. 115. - Nelops . Linn. Labro melope. Lappanu dí Niuri.

116. Mixtus . Linn. Labro mischio. Lappanz Paronessa.

11\%. Meruk . Linn. Labro merlo, Turdo a' Arcw. 118. Julis . Linn. Labro julide . Viola a Mese sima Vidiola.

119. - Cynedus . Linn. Labro cínedo. Pizzi di Rè, ò pesce di Rè. a Catania Jodiolu? a Mrssima Vidiola impiriali.

120. Bimaculatus. Línn. Labro bimacchiato. Pitcmeda impiriali. 


\section{( 22$)$}

121. Labrus Turdus Linn.

Labro tordo:

Turdo, o Merlu.

122.

Reticulatus. Linn.

Labro reticulato .

Turdo .

123. Guttatus. Linn. Labro cocciolato .

Turdo

Stizziatu.

- 121

Ciavolus. Raf. car. sp. 109. Labro ciavolo.

Sciavolu, Sciaula, Sciaura, ò Ciaulu .

* 125. Lappanus. Raf. car, sp. 106. Labro lappano. Lappanu di portu, a Catania, e Messina Lap. paru, ̀̀ Lappara.

* 126. Donzella . Raf. car. sp: 105. Labro donzella . Donzella, Dunzedda, ò Pizzi di Rè impiriali.

* 127. Chorophtalmus . Raf. car. sp. 108.

Labro verdocchi . Lappanu Occhiu-Virdi, ò Turdo .

* 128. Pittima . Raf. car. sp. 9\%. Labro pittima. Pittima, ò pesce di pietra.

* 129. - Pittimoides . Raf. car. sp. 93. Labro pittimoide. Pittimedda.

7130. Lappanoides . Raf. car. sp. 107. Labro lapa panoide”. Lappanu Turdo.

131

131. Zittus. Raf. car. sp. 103. Labro Zitto. Turdo zittu.

132. Zittoides. Raf. car. sp. 102. Labro zittoide: Turdo zittu impiriali a Catania Zittu .

* 133. Verdolidus . Kaf. car. sp. 91. Labro verdolido. Verdolidu.

* $3 t_{0}$ Macrostomus . Raf. car. sp. 95. Labro ma-. extomo. Trumbetta. 


\section{( 23$)$}

* 135. Labrus Fucii . Raf. car. sp. 100 Labะo dei fuci. Pittimedda d'Arca .

136. Xantherythrus. Raf. car. sp. 111. Labro gial. lorosso . Papagaddu impiriali .

187. Porcus . Raf. car. sp. 97.

Labro porco. Turdo percu .

* 158. Ocultus-perdix Raf. car. sp. 104. Labro occhiopernice . Pittimedda occhiu pirnici.

* 139 Leo . Raf. car. sp. 96. Labro leone. ce leone.

* 140.- 1mperialis . Raf. car. sp. 112. Labro imperize le. Lappanu impiriali.

* 141. Chrysostoma . Raf. car. sp. 98. Labro bocca. d' oro. Bocca-d'oro, d Lappanu Bocca. d'oro . a Catania Pizzoru .

* 142. Caliophthalmus, Rafo, car. sp. 39. Labro bel. locchio . Occhiu-beddu .

* 143. - Melanotus . Raf. car. sp. 110. Labro dorso. nero . Lappanu di niuri.

* 144. Luvarus. Raf. car. sp. 101. Labro luvaro 。 Lappanu luvaru, ò russignu.

* 145. - Marmoratus . Raf. car. sp. 113. Labro marmorino . Lappanu marmurinu .

* 146. Mendovella. Rafo app. sp. 13. Labro mendovella. Mendorella, ò Menduredda. in Trapani. Ucchiatedda.

147. - Cettii . Raf. app. sp. 14. Labro di-cetti . Viola impiriali. 
148. Spicara Flexuosa. Raf. car. gen. 3j. sp. 140. Spicara flessuosa. Spicara.

149. Sparus dentex. Linu. ' Sparo dente. Dentice, $\boldsymbol{P}_{e}$ sce dintatu a Messina .

150. - Auratos. Linn. Sparo Orata . Dentice arata, a Catania Zuratu, a Saci Vidichiu, ed a Malta Aurada.

151. Sargiu. Linn. Sparo sarago Saragu, o Saracu.

152. - Puntazzo. Linn. Sparo puntazzo. Saracı Pizzudo.

153. - Smaris. Linn. Sparo Smiricie Minola inpiriali.

154. - Mana . Lim. Sparo Mendola. Minola.

155. - Melanuus . Linn. ( sp. oblada Lac.) Sparo occkiata. Occhiata a Messina Biata

156. Hurta. Linn. Sparo Urta. Praì impirialio a Catania Pauru Ricali.

157. - Pagrus . Linn. Sparo pagro. Praì, ò $P_{a}$ gura. a Messina, od a Catania Pauru.

168. - Erythrinus. Linn. (sp. pagel. Lac.) Sparo pagello. Lwvaru. a Messina Pragagnanu. 159. - Boops . Linn. Sparo boga . Vuopa a Catania Balajola.

160: - Salpa . Linn. Sparo Salpa . Sarpa, Salpa, ò Saupa:

161. Cantharis . Linn. Sparo cantaro . Ciuciastra, - Sarpa impiriali. $16 \%$ 


\section{$(\div 25+\div$}

162. Sparus mornyras Linn. Sparo morniro.

Ajuta im. : piriali.

153. Chromis Linn. Sparo cromide. Monacedda.

164.

Orphus . Linn.

Sparo orso . Mupa im-. piriali.

165. Bogaraveus. Lac. Sparo bogararco - Fuo pa impiriali, a Catania Pampini.

166. - Claviera . Lac, Sparo claviera. Minolinda : 167. Lividus . Lac. Sparo livido. Monacedda impiriali.

168. _- Massiliense . Lac. (a) Sparo marsigliese . Asineddu di. Varru

* 169. - Auratoides. Kaf. car. sp. 139. Sparo Oratina. Arata.

170. Mupa ! Raf. car. sp. 129. Sparo mupa. Mupa.

* 171. Adottus . Raf. car. sp. 138. Sparo Adotto . Adottu, ò Dottue.

* 172. Gibbosus. Raf. car. sp. 126. Sparo gobbo . Paulu incoronatu . ò Praù impiriali nel ral di Mazzara, Pague curunatu nel val demone, c Croz a nel val di Noto.

173. Gajolus. Raf. car. sp. 127. Sparo gajolo. Gajolu, Ajola, sajola, ì Ajuolu . a Catania Ajulo.

(6. a) Questo Sparo avendo un appendice squamoso frà le ale toracine, dovrebbe forse formare us genere particolare, che chiamerei Merolepis. 


\section{$(20)$}

* 174. Sparus Varatulus . Raf. car. sp. 128. Sparovaratulo Varatutu, Varatru, Varctula, Guaratulu, o Oratulu,

*. 175. Trifasciatus . Raf. car. sp. 135. Sparo trifas. ciato. Saragu fanfuru .

* 176. - Zippolus . Raf. car. sp. 137. Sparo zippolo . Zippolu, cippola, ò Zipola a Trapani í anuia . - 177. Polynymus. Raf. car. sp. 134. Sparo polni- mo. Asineddu, nella sua adolescenza Riton. $d u$, nella sua piccolezza Macaronecidu . a Trapani Macchictlu.

* 178. Minutus. Raf. car. sp. 133. Sparo milluto. Monaceddus:u.

* 179. - Sparulus . Raf. car. sp. 130. (non Lac.)

Sparo sparulo. Sparajone, ò sparaglione. * 180. Sparlotus . Raf. car. sp. 132. Sparo Sparioto . Sparlottu.

* 181._- Ophthalmicus . Raf. car. sp. 131. Sparo Oc. chiatella. Occhiata impiriali.

* 182. - Scirenga. Raf. car. sp. 156. Sparo Scirenga . Scirenga.

183. Diplodus annularis. Raf. app. gen, 6. (Sparus annularis Linn., Sparus sparulus Lac. ). Diplodoannellare. Sparajone impiriali.

" 1S1. Dipterodon ruber. Raf. car. sp. 125. Dipterodo rosso. DIunacedda russa.

- 185. Gonenion serra. Raf. car. gen. 39. sp. 143. Gonenio serra. Pesce serra. 


\section{$(2 \pi)$}

186. Mullus rüber. Lin.: , Ml ulleto rossi.

Riviglia di fange $u$

18\% - Surmuletus. Liun. Millelo triglia.

Trislia di solo a Palermo, e'Triglia di Gramigna a Messina.

* 158. - Fuscatus. Raf. car. sp. 91. , Nulleto foschic. cio. Triglia d' arca, ò di niuri nel val di Mazzara, Sparagalaci a Messina, Catania, c nel val di Noto.

189. Apogon. Linn. (Apogon ruber Lac.) Mulleto sbarbato. Triglia slarbala.

190. Scorpena . ( a ) rascassa Lac. (Sc. porcus Linn.) Scorpena rascassa. Scrofani, ì Scorfanu,

a Catania Scaropali.

192. - Scrofa Linn. Scorpena scrofa. Scrofana . a Trapani Cepola, o Cipola a Catania Scazupuli.

193. Massiliense . Lac. (Cottus massiliense. Linn.) Scorpena marsigliese. Scrofune impin riali .

191. - Notata. Raf. car. sp. 83. Scorpena macchiata. Scrofaneddu.

TER.

( a ) 2u sto genere si dovrì forse più convenevolinente raguagliare nell'X111. ordine dei Percidi, a motivodei suoi Upcroli spirosi, ò appendicolati: 


\section{$(28)$}

TERZA SEZIONE.

GLI ORTONOT1 :

Corpo allungato, coniro, lonceolato, ò cilindrico, molto più lungo, chie alto, e col dorso dritto, ò appenä curvo.

\section{XV111. ORDINE. 1 DACTIPL1.}

Corpo conico, dei raggi sciolti, ò riuniti vicino ad ogni ala pettorale.

195. Dactylopterus pirapera . Lac. (Trigla volitans. Linn.)

Datilotero volante. Galinedda, o Pesce falcone. 196. Trigla lyra . Linn Triglia lira . Furcata . 197.- Hirundo Linn. Triglia rondinella . Fagiana impiriali, ̀̀ Pesce redenune.

135. - Gurnardus Linn." Triglia brontola . Tigicgu, ò Tigiega .

199. - Cuculus . Linn. Triglia cncülo e Ċuccu, o Coccidlue.

200. _- Lostoviza, Lac. (Tr. adriatica Linn.) Triglia lastoviza. Pesce papa.

201. Cavillone . Lac. Triglia cavillone. Marteduz zu impiriali.

* 202. Tagianus . Raf. car. sp. So. Triglia fagiano. Fagiana, ò Pesce Fagianu. a Catania Faranz, 203. Corvus . Raf. car. sp. 81. Triglia corvo • Pesce corvu.

201. - Gorotus - Raf. car. sp. 82. Triglia gonota • Mcatidduzzu, ̀̀ Martelluz̃o.

305. Peristedion chabronterus .. Lac. Peristedio chabronte. Trafino impiriali. 206. 


\section{( 29$)$}

206. Octoaus olosteon Raf. app. gen. 7 . (Trighia catapuracta.

Linn. Pcristedion malarmat. Lac.) Octono

olosteo. Pesce Trafino, ò fulcata impiriali ..

201. Lepadogaster govani. Lac. Lepodogastro di guano.

Ampiscica impiriali.

\section{X1X. ORDINE . GL1 ECIIENEIDI .}

Corpo conico, una piastra lamallosa sopra il capo, senza ; raggi sciolti, o riuniti vieino ad ogni ala pettorale.

208. Echeneis remora . Linn. Echeneo remora . Ams piscica.

- 209. 1mperati. Raf. (Echeneis mediteraneus. Raf. car. sp.) Echeneo d' imperati . Anpiscica .

\section{ORDINE . 1 CORIFENIDI :}

Corpo coníco, senza piastra lamellosa sopra it capo, re raggi sciclti, o riuniti vicino ad ogni ala pettorale.

* 210. Coryphena imperialis . Raf. car. sp. 84. Corifena imperiale. Cappune impiriali.

211. Hippurus. Linn. Corifena ippuro:

Cappune .

2 212. - Hippuroides Raf. ( Lepimphis hippuroides Raf. car. sp. 86.) Corifena Cappone. Pesce Cappone, ò Cappune. a Siracusa, Agosta, ect il val di Noto Lambacu, ̀̀ Lampugu .

213. Coryphena pompilas Linn. Corifena pompiló.

Piliu, ò Lambuco. 
214. Coryphena novacula Linn. Corifena novacula . Pettine .

* 215. - Lineolata . Raf. car. sp. 85. Corifena lineolata . Pettine impiriali.

216. Lepimphis ruber. Raf. car. gen. 28. sp. S7. Lepirfo rosso. Pesce pettine, ò Monaceddu Masculu.

+ 217. Cottus gobio. Linn. Cotto ghiozzo. Gozzu. 213. Gobius aphia . Linn. Ghiozzo afide. Urgiuneddu: 219. Maganellus. Linn. Ghiozzo pagmello .

Urgiune, Urgone, ̀̀ Gorgione a Palermo, e nel val di Mazzara. Mazzone a Messina, c nel val di Noto.

220. Cruentatus . Lim. Ghiozzo macchiato. come il precedente.

221. - Nigrofuscus . Lac. (Gob. bicolor. Linn.)

Ghiozzo nerofosco. Urgiune di fortuna.

222. - Niger . Linn. (Gob. boulerot. Lac.) Ghozzo nero. Urgiune di niuri.

223. Rubens - Rafo car. sp. 88. Giozzo rossigno.

Urgiune sanguignu.

224. - Gorgione. Raf. car. sp. 89. Ghiozzo gorgione. Gorgione, ò Urgiune di fangu.

\section{ORDINE. GLI ISTIOFORIDI.}

Corpo, e mascelle allungate, Ale toracine con i raggi riuniti senza membrana intermedia .

* 225. Tetrapturus belone. Raf. car. gen. 41. sp. 145. Te. tratturo aguglia. Agıija,ò Aguglia pelerana. 


\section{(31)}

\section{XX11. ORDINE I CEPOLIDI.}

Gorpo molto allungato, lanceolato, ò Serpentiforme; avendo nessun' ala di meno.

226. Cepola tenia. Einn. Cepola tenia . Bandiera impiriali. 227. Rubescens. Linn. Cepola rossigna. Bandie. ra russigna.

298. Trachyptera. Linn. Cepola trachittera . Bandiera impiriali.

* 299. - Marginata. Raf. car. sp. 14\%. Cepola margina ta . Bandiera impiriali.

230. Lcpidopus govani Lac. Lepidopo di guano a Pe? sce maristu.

\section{XX111. ORDINE. 1 GINNETRIDI .}

Nessun' Ala anale.

* 231. Argyctius quadrimaculatus . Raf. car. gen. 42. sp. 146, Argittio quattromacchie.

Pesce marishe. Tavala 1. fig. 3.)

* 132. Cephalepis ectomaculatus . Raf. app. gen. 8. sp. 15.

Cefalepio ottomacchie : Pesce mavistu.

XX1V. ORDINE.

TERZA DIVISIONE.
1 GINNURINI.

\section{GLI ADDOMINALI。}

Ale inferiori situate sotto il ventre, 0 posteriormente alle ale pettorali. 


\section{$(+32)$ \\ PRIMA SEZIONE . \\ I TOSSOGASTRA :}

Corpo ovale, ò ellitivo, col dorso, ò ventre curvo

XXV. ORDINE." i POLLINEMIDI .

XXV1. ORDINE. 1 SALMONIDI:

Due ale dorsali, la seconda adiposa, senza raggi sciolti,

o riuniti vicino alle ale pettorali, dorso, ne ventre carenato.

233. Salmo tirus. Raf. car. sp. 148. Salmone tiro. Ti.

ru. impiriali.

$+* 231$.

Cetti . Raf. app. sp. 16. Salmone di Cetti .

Trulta di Sciume.

235. Osmerus sanrus . Lac̈. (Salmo saurus Linn.) Osinero

sauro. Tirk ai solo, ò Trutta di mare.

\section{XXV11. ORDINE . 1 CLUPIDI.}

Dorso, o ventre carenato .

236. Clipea alosa. Linn. Clupea alosa . Alosa, a Catania Alockio.

287. - Sprattus.. Linn. Clupea sardina.'Sarda, ò sardella.

238. - Encrassicolus Linn. Clupea acciuga. Alice, ò anciource, ò anciover .

239. - Allecia . Raf. car. sp. 150. Clupea allecia . Allecia, Alachia, ò Allacia.

210. - Atherinoides . Linn. Clupea argentina

$C u-$ runedda surda.

XXV111. ORDINE.

1 CIPRINIDI.

Senza raggi sciolti, o rimiti vicino alle ale pettorali, e senz' ale adipose, dorso, ne yentre carcuato. $\$ 241$. 
- 211. Mugil cephlalus . Linn. Mugile cefalo. Molettre nel val di Mazzara, Cefalu nel val demone, c Lampune nel val di Noto.

1. Var, cestreo . Raf. app. sp. 17 .

Betto var. cestreo . Calvinu nel val di Maz. zara, Lustru nel val Demone, e di Noto.

2. Var. myxone , Raf. app. sp. 17.

Betto var, missone. Cirinu .

3. Var. chelone . Raf. app. sp. 17. Detto chelone. Cefaiune.

242. Cyprinus tinca. Linn. Ciprino tinca. Teng $a d i$ Sciumi .

† 243. - Carpio. Linn. Ciprino carpa. Carpiune. t. 244. - Auratus. Linn. Ciprino dorato . Pesci rus w su, ò Pisci indianu.

\section{SECONDA SEZ1ONE. GLI ORTOGASTR1.}

Eorpo conico, cilindrico, ò lanceolato, col dorso, ell i! ventre dritto.

\section{ORDINE. I POLITTERINI. XXX. ORDINE. 1 SAIRIDINI.}

Senza spiragli, diverse piccole ale sopra, e sotto della coda, mascelle rostrate.

Q15. Sayris recurvirostra . Raf. car. gen. 45. sp. 160. ( Scombresox camperi Lac. ) Sairide rostrocurro . Cristaredda. a Messina Cristardedda. 


\section{(31)}

* 216. Sayris hians. Raf. car. sp. 161. Sairide rostraperto . Cristaredda, Tristaredda, ̀ Ristardedda.

- 147. Serrata. Raf. car. sp. 159. Sairide serrata . come la precedente.

- 218. Maculata. Maf. car. sp. 162. Sairide macchiata. Cristareddo impiriali.

\section{XXX1. ORDRNE . GLI ESOC1DI.}

Due, o tre ale dorsali, Mascelle rostrate.

219. Sphyrena spet Lac. ( Esox sphyrena Linn.) Sfircinaluzzo. Aluz:u, ò Aduะzu, a Catania Loะ̃o .

250. Esox belone. Linn. Aguglia comune . Aguglia, ò Aguja, a Catania Auguglio .

* 251. - Imperialis . Raf. car. sp. 157. Aguglia imperiale, Aguja impiriali; a Catania $A u$ guglio reale.

- 952. Sưdis hyalina. Raf. car. gen. 44. sp. 158. Sudia jali. na. Aduzzu impiriali, a Catania Loz

\section{XXX11. ORDINE . I NOTACANTINI}

\section{XXX11. ORDINE - (a) 1 CENTRISCHIN1.}

Corpo ricoperto di una corazza, capo prolungato in un mu* so allungato.

253. Centriscus Scolopax. Linn. Centrisco beccaccio.

$$
\text { Irumbina. }
$$

XXXIV.

(a) Questo Ordine sard probabilmente meglio collocato nel: a prima Sezione degli addominali, avendoil dorso un pococurvo. 
XXXIV. ORDINE.

XXXV, ORDINE。
1 LORICARINI.

1 SILURIDI .

Corpo conico, senza cornzza, no piastra, capo schiacciato, ed allargato .

254. Inacroramplosts cornutus . Lac. (Silurus cornutus Linn.) Macranfo cornuto. Caniscu impiriali.

\section{XXXV1: ORDHE. GLI ESOCLTINI}

Corpo conico, senza enrozza, ne phintra, capo pih,

o. meno schlacciato ma senpre stretto.

255. Exocetus exiliens "Linn. "Esoceto volante .

fits cileddu impiriali.

- 256. - Heteruruss. Raf, car. sp, 156. - Esoccto inu- guale . Ancileddu, Angiletbu, Ancireddu, Ancilune, Redenune, e Renidinune, a Messina Grillo, ¿̀ Griddu.

* 25\%. Tirus marmoratus . Raf. car. genen. 43. sp." 119. Tiro - marmorato : Tiru, ò pesce tiru. .

- 258. Myctophum punctatum . Raf. app. gen. 9. sp. 18.

Mittoso puntato. Ancioure impiriali, o $C a$. nischeddu. Tavola . 2. fig. 5.

259. Argentina sphyrena. Linn. Argentina sfirena - Curu. nedda, a Catania Corinella, a Messina Curinedda.

260. — Imperialis - Raf. car. sp. 154. Argentina im. perialc . Curanedda impiriali.

2 361. - Aphia. Raf. car. sp. 155. Argentina afia . Nunnata, a Messina Majatica. 
+ 202. Atherina hepsetus. Linn.

Alterina ossetto.

Curue nedda marzoja. a Catania Corinella di Sciume.

- 205. Coroneda . Raf. car. sp. I51.

Aterina cors. neda. Coroneda, ò Curanedda mazzaruta.

* S61. - Lattarina . Raf. car. sp. 159. Aterina latari. na. Curunedda lattarina.

* 9.5. Nunnata . Raf. car. sp. 153. Aterina numata. Nunnata, ò Nunnaledda.

XXXV11. ORDINE. GLI AMIDI.

XXXVI11. ORDINE. I BUTIRINIDI.

XXXIX. ORDINE. I CULUMBRINIDI.-

XL. ORDINE - GLI OLOSTOMIDI.

QUARTA DIVISIONE. GLI APODI.

sezza Ale inferiori.

\section{PIRIMA SEZ1ONE. 1 MACROSOMI.}

Corpo allungato, conico, lanceolato, ò cilindrico .

\section{XL1. ORDINE. I SIGNATIDI.}

Corpo molto allungato, angoloso, ì cilindrico, ri.

coperto di una corazza, bocca all' estremita

di un muso allungato, tuboloso,

e senza denti.

206. Typhle hexagonus. Raf. car. gen. 17. (Syngnathus tiphle
Linu. Lac.)
Tifle esagono .
Gieju .

267. Siphostoma acus. Raf. car. gen. 18. (Syngnathus pela. gicus . Linn, ) Sifostoma aguglia. Agu• jcenda, ò Trumbettina, ò Serpuzac di marts 
* 205. Siphostoma Viridis. Raf. app. sp. 19. Sifostoma verde.

$$
\text { cone il precedente. }
$$

869. Hippocampus heptagonus. Raf. car. gen. 19. ( Syngzathus hippocampus Linn.) 1pocampo caval. letto. Cavadduzzu marinu.

270: Syngnathus punctatus. Raf. app. gen. 10. sp. 20. Signatto puntato. Trumbettina.

* 971. Nerophis maculatus . Raf. app. gen. 11. sp. 21. Nerofide macchiato. Spingola : di Mari. Tavola 2. fig. 4.

\section{XL11. ORDINE. \ TRIURIDI ..}

\section{XL111. ORDINE . T TRICHIURIVI. XLIV. ORDINE. I GINNOTINI.}

Corpo cilindrico, serpentiforme, e viscoso, alcuro Ale di meno.

272. Carapus acus . Raf. app. gen. 12. (Gymnotus acus Linn.) Carapo aguglia. Ancidduzza..

273. Ophisuras serpens Lac. (Murena serpens. Lin.) Ofi. suro serpente. Ancidda serpa, ò Serpa di niuri.

- 374. Oxyrus rermiformis Raf. car, gen. 20: sp. 41. Ossiuro

$$
\text { verme. Vermuz } z \text { di Mari. }
$$

\section{ORDINE. GLI ANGUILLIDI.}

Corpo cilindrico, e. Serpentiförme, nessun' ala di meno.

- 275. Anguilla Vulgaris. Raf. app. gen. ( M urena anguilla.

Linil.)

Anguilla comane . Ancidda. 
Aguilla vulgaris

\section{( $(38)$}

1. Var. marina Detta var. marina.. Ancidda di mari.

2. Var. fluviatilis. 'Detta di fume..

Ancidda di Sciumi .

3. Vai. lacustus. Detta di lagho.

Ancidda di Biviere.

276. Congér. Raf. (Murena conger. Linn.) Anguilla -grongo: $\because$ Grungu.

277. Myrus Raf. (Murena myrus Linn.) Anguilla miro. $\because$ Grungu di Solu.

\section{XLVI: ORDINE. GLI OFIDINI.}

Corpo lanceolátó c compressiśsimo, nessun ala di meno. 278. Ophidium: maculatum. Raf. (Ophidium barbatum Linn.)

Ofidio macchiato. Bandiera imperiali. 279. Physocephalum Raf. car. $\$$ P. 45. Ofidio gono - fiato. Bandiera vranca.

280. Chysocephalum. Raf. car. Sp. 46. Ófidio ca. pod'oro. Bandiera di niuri.

281.- Punctatum. Raf. car. Sp. 47. Ofidio punteg griato . Bandierina di Solu.

282. - Fulvescens. Raf. (Ophidium imberbe Linn. .

Ofidio fulvastro Bandiera giarnusa.

283. Ammorlytes cicerellus. Raf. car. Sp. 52 Amodito cicercllo. Cicerellu, Cicireddu, ò Cicirello. 284. Scarcina argyrea. Rẫ. car. gen. 21. Sp. 48. Scarcinea argentata. Scarcina, a Messina Spatole: 985. Punctata. Raf. car. Sp. 49. Scarcina panteg. giata. Scercinedda 
- 286. Scarcina Quadrimaculata. Raf. car. Sp. 50. Scarcina. quatromacchie. Scarcina di forte.

* 287. Imperialis Raf. car. Sp. 51. Scarcina impe. riale. Scarcina imperiali...

\section{OIRDINE . I Z1FIDI.}

Corpo conico, ò lanceolato, nessun' ala di meno, Muso colla mascella superiore molto prolungata, ì Spinosa. 288. Xypluias gladius Linn. Zifią Spada. I'esce Spada, ̀̀ Pisci Spalu.

\section{ORDINE, COMEFORINT。}

SECONDA SEZIONE, I BRACHISOMI

Corpo corto, sferico ovale, ’̀ cllittico.

\section{ORDINE. I STROMATINI .}

Corpo molto compresso, nessun ala di meno, mascelle norossee, e per lo più senza denti.

289. Stromateus fratola Linn. Stromatce
la imperiali, ò Fialulu.

* 290. Luvarus imperialis Raf. car. gen. 25. Sp. 53. Luvaro imperiale. Luiaru impiriali, a Catania Luvaru reale. Tar. 1. fig. 1 .

\section{ORDINE G GII OSTRACIDI .}

Corpo ricoperto di una Corazza, nessun' ala di meno, ma scelle non oşsee, mạ dentate.

231. Ostracion nasus. Linn. Ostracio naso. Pesce Soddu, ò Pesce porcu. 


\section{$(40)$}

992. Ostracion Agonus. Raf. app. 8p. 22 Os tracio sferic Pesce porcu.

\section{ORDINE . GLI ODONTIN1 .}

Nessun' ala di meno, mascelle ossce

293. Tetrodon hispidus. Lin. Tetrodo ispido. Meringianu .

294. Diodon echinus. Raf. app. sp. 23. Diodonte echino. Pesce rizau.

295. Orthragus luna Raf. (Tetrodon mola Linn.) Ortrago luna Pesce luna, ò Tamburru imperiali.

296. Oblongus. Raf. car. gen. 15. sp. 42.

Ortrago bislungo. Tamburru coronatu. 297. Diplanchias mola. Raf. car. gen. 16. sp. 43. Diplanchia mola. Pesce tamburru.

III. ORDINE. GLI ORBIDI.

\section{SEECONDA SOTTO CLASSE. GLI ATELINI.}

Branchie spreviste di opercolo, ò Membrana branchiabe, ò di ambidue.

\section{PRIMA DIVISIONE. 1 POMANCHIDI.}

Branchie con opercolo, ma seña membrana branchiale.

L111. ORDINE. STERNOTT1DI.

LIV. ORDINE. 1 STÚRIONIDI .

Córpo allungato, nessun' ala di meno, l' ale inferiori adklominali, muso allungato. 
+ 293. Sturio vulgaris. Raf. àpp. gen. 13. (Acipenser Slurio Limn.) Storione comune. Sturiune, a Messina Iruictu.

\section{ORDINE . 1 COGRIDI .}

Corpo lancculato, sprovisto di diverse Ale.

* 299. Cogrus maculatus. Raf. car. gen. 46. sp. 163. macchiato Bandicrina.

Cogeco

\section{SECONDA DITISIONE . GLI OMNANCHIDI.}

Branchie proviste di membrana branchiale, ma senza opercolo .

$$
\begin{aligned}
& \text { LV1. ORDINE. } 1 \text { MORMIRIM . } \\
& \text { LV1I. ORD1NE. } 1 \text { CHIMERIN1. } \\
& \text { Corpo conico, ale inferiori addominali. }
\end{aligned}
$$

* 300. Pieccephalus adherens. Raf. car. gen. 47. sp. 16\%. Pie. cefalo aderente. I'esce campiscica nel val ti Mazzara, Pesce diazalo, ò Anpiscicaloru a Messina, Catania, ecl il val di Neto. a

\section{LV111. ORDINE. I BALISTINI.}

Corpo compresso, una sola ala inferiore, e toracina.

* 301. Balistes annularis. Raf. car. sp. 41. Balista anncllata. Pesce porcu.

302. Capriscus porcus. Raf, app. gen. 14. Capurco porco. Pesce porcu. 


\section{(42) \\ LIX. ORDINE, 1 LOFIDI :}

Ale inferiori jugulari, o situate sotto la gola 303. Lophius piscatorius Linn. Lofio pescatore

Pescas: trice, Maga, ò Giurana di mari.

\section{ORDINE. GLI ECHELINI.}

Corpo cilindrico, senza ale inferiori .

- 304. Echelus macropterus. Raf. car. gen. 48. sp. 765.

Echelo macrottero. Grungu di niuri.

* 305. - Nebulosus. Raf. car. sp. 166. Echelo nu. voloso Grungu di fangu.

* 306. Oxyrinchus. Raf. car. sp. 167. Echelo masino. Afferre Vacche di mari.

207. Microphthalmus. Raf. car. sp. 168. Echcle piccolocchio. Grungu d' arce .

* 308. Echclus rufus. Raf. car. sp. 109. Echclo fulvo

Spari matrimoniu di mari.

309. Auratus. Raf. car. sp. 173. Echelo indorate Grungu di solu, ò a induratu.

- 310. Gruncus. Raf. car. sp. 170. Echelo grunco Grungu di pictra.

* 311. Cinciara. Raf. car. sp. 172, Echelo ciuciara: Ciuciara.

312.- Punctatus. Raf. car. sp. 171. Echolo pun? teggiato. Ciuciars imperiali.

\section{IXI. ORDINE. 1 CLOPSIDINI.}

Corpo Cilindrico, senza ale inferiori, ne pettorali.

* 313. Chlopsis bicolor. Raf. app. gen. 15. sp. 2t. Clopride bicolore. Ancidda impiriali. Tav, 2. fig. 1 e 


\section{(43)}

6 314. Nettastoma melanura . Raf. car. gen. 49. sp. 174.

Nettastoma codanera. P'esce Ocel, ò Pizull d' anatra.

\section{OIDINE, 1 ZITTERINI .}

Corpo lanccolato, compresso, senza ale inferiori, ne pettorali, ne caudali .

315. Xypterus inperati . Raf. app. gen. 16. sp. 25. Zittcro d'imperati. Spada di mari, ò Porte bandiera.

\section{TERZA DIVISIONE. 1 GINNANCHIDI.}

Branchic sproviste di Opercolo, e di membrana branchials.

\section{PRIMA SEZIONE. 1 DIPLANCHIDI .}

Eorpo provisto d' ale, e di un' apertura branchiale da ogni lato .

\section{LX111. ORD1NE. I MONOTTER1D1.}

Una sola Ala.

* 316 Pterurus flexuosus. Raf. app. gen. 17. sp. 26. Co. dala flessuoso. Casentula di mari, ò Morin na pollina.

\section{IXIV, ORDINE. 1 DALOFIDINI .}

Un' ala dorsale, ed un'ala anale, nessun' altra.

* 317. Dalophis Serpa. Rafo car. gen. 51. sp. I77. Dalofia de serpa. Serpa di mari.

* 318. - Dimaculata. Raf. car. sp. 178. Dalofide due mnechic. Sryuzza taecbiata. 


\section{$(44)$}

\section{ORDINE. I MURENIDI .}

Un' asia dorsale, on' ala anale, ed un' ala caudale riunite assiemc .

319. Murena helena Linn. (Gymnothorax helena . Bloch. Murenophis Lelena. Lac.) . Morena comune Murena, ò Murina.

$\$ 320$. Variegata. Raf. car. sp. 175. gata. Irzrina d'arca.

* 391 Punctata Paf. car. sp. 176. Murena ponteg. siata. Garajune, Guaragilione, Marajone, o II wina impiriati, a Catania Quadaruni.

\section{SECONDA SEZIONE. I POLIANCHIDI:}

Corpo provisto d'ale, c di più molte aperture branchiali da ogni lato.

\section{LXV1. ORDINE I CHONDROTTERI.}

Corpo allungato, ma non schiacciato, da tre a sette aperturo brancliali da ogrni lato del collo, nessun' ala di me. no, fuori alcune rolte dell'anale, le inferiori addo. minali.

* 329. Dalatias Sparophagris. Rap. car. ggen. 2. sp. 26. Dahtia sparofago. Níngia luvaru.

* 393. - Nocturnus. Raf car. sp. 27. Dalatia notturno. Pesce nolle, ò Pesce di notti.

381. Carcharias lamia. Raf. car. gen. 1. (Squalus carcharias. linn.) Carcaria lamia Pesce.Cane, Imbestinu, ò Lamia. 


\section{( 45$)$}

325. Charcharias Glaucus. Liaf. (Squalus glaucus Lac). Carcaria gylauco .

$V$ erdescu .

$*$ Taurus. Raf. car. sp. 21.

Carcaria tauro :

Pesce tauru.

327. Ileptranchias cinereus. Raf. car. gen. 7. ( Squalus cine. reus. Lac.) Etranchia cincreo. Pesce Anciovu.

* 325. Alopias macrourus. Raf. car. gen. 6. sp. 31. Alopia coulalunga. Pesce surciu.

* 329. Isurus oxyrinchus. Raf. car gen. 4. sp. 29. 1suro musaciato - Pesce tundu.

* 330. Spallanzani. Raf. app. sp. 27. Isuro di spallanzani. Pesce Cane,

* 331. Cerictils macrouras. Raf. car. sen. 5. sp. 30.

Cea - rittio codalunga. Pesce diarolu, ò Pesce

Comula.

* 398. Caniscus. Raf. app, sp. 23.

Cerittio canisco: Caniscu, ò Pesce caniscu .

333. Squalus accanthias. linn. Squalo accantia.

Ujatzo impiriali.

334. - Spinay. linn. Squato spinoso. Pesce notte impiricali.

* 395. - Uyatus . Raf. car. sp. 33. Squalo uyato. Tyatu, ò Ujatu, a Catania Pisci maz:i. 336. Oxynotus centrina. Raf. app. gen. 18. Ossiñoto cene trino. Pesce mazzapani, ò Marzapani. 337. Rina Squatina. Raf. car. gen. 11. Rina Squatina. Squadru. 
338. Pristis antiquorum. Lac. Pristo pesce scgla . Pe. sce Serra.

339. Aodon comutus. Lac. Aodonte comato. Diavolu cors nutu, ò Pesce catalia.

340. Etmopterus aculeatus Raf. car. gen. 10. sp. 34. Em. mottero spinoso. Diavulicchiu di mari.

341. Tetroras angiova. Raf. car. gen. 3. sp. 28. Te= trora angiova. Angiova, Angiovo, ò Sturiuni di mari.

312. Galcus catulus. Raf. car. gen. 8. ( Squalus catulus linns)

Galco gatto. Gullucciu, ò Bestinu .

343. - Stellaris. Raf. (Squalus stellaris lin.) Galeo stellato. Gatlucciu imperiali.

344. Caniculus Raf. (Squalus caniculus lim.)
Galco cane. Gaitupardu.

345. Mustellus. Raf. (Squalus mustellue linn. Ga. leo mustello. Palumbu, ò Pesce palumbo a Siracusa Canigrossu.

316. Melastomus. Raf. car. sp. 32. Galeo boccane ra. Bocce d' infernu, a Catania Palumba passanita .

31\%. Asterias. Raf. ( Squalus asterias Linn.) Galeo asteriade. Gatlupardu imperiali.

318. _ Vulpecula . Raf. (Squalus vulpecula Linin).

Galeo volpino. Pesce sorciu imperiali, o Cudalonga.

619. Sphyrna zygena Raf. app. gen. 19. ( Squalus zygeria Liun ). Sfira zigena. Lestone, ò Peo sce giudet: 


\section{$(47)$}

350. Sphyrna Liburo. Raf. (Squalus tiburo Linn.) sfirna tiburone. Magnusı, ̀े Сrozaa.

351. Hexanchus griseus. Raf. car. gen. 9. (squalus griseus

Lae. Essanclio bigio. Anciovu impirialio

\section{LIVIII. ORDINE. I PLATOSOMI.}

Corpo schiacciato, dieci aperture branchiali al dí sotto cinque da ogni lato, l'ala anale di meno, e qualche voita le dorsali, e caudali, ale pettorali orizontali, le, inferior $\overline{\text { i }}$ addominali .

352. Raja oxyrynchus. Linn. Raja musacuto. Pigara liscia, ò Picara lizza, a Catania Raja. . 353. Miraletus. Linn. Raja miraleto. Pioura quattrocchj .

35\%. Fullonica. Linn. Raja fullonica. Picara magnusa, a Catania Ruvetu.

355. Clavata. Lina. Raja chiodata. pietrosa :

* 356. Ciodera Raf. car. sp. 35. Raja ciodera.

\section{Ciodera .}

5 357. Pigara. Raf. car. sp. 37. Raja pigara.

\section{Pigarb latina.}

* 358. Macrorynchus . Raf. car. sp. 38. Raja ma solungo Pigara scapucina .

* 359. Fenestrata. Raf. car, sp. 36. Raja fenestrata. Pesce scillarce.

360. Rubus. Linn. Raja spinosi. Pigard spia nose, ò ruretu. 
361. Leiojatus pauduratus. Raf. car. gen. 12. sp. 39. Jisbato pandurato. Pesce viulinu.

302. Aquila Raf. (Raja aquila Linn.) Lebato aquila. Pesce aquila, ò Aquilu di mari a Messina Pigara liscia.

363. Torpedo ocellata Raf. app. gen. 20. sp. 29. (Raja Torpedo Linn.) Torpilla occhiata Tremola scacchiate, ò vechiuta.

864. Immaculata. Raf. app. sp. 30. (Raja torpedo. var. Linn.) 'Torpilla liscia. Tremola sen:a tacche.

365. - Punctata. Raf. app. sp. 31. (Raja torpedo var. ' lim.) Torpilla puntegrgiata. Tremoia piz zicata.

536. Dipturus batis. Raf. car. gei. 13. (Raja batis Linn.)

Disturo batide. Bastunanagha impiriali. 367. Mobuli auriculata. Raf. app. gen. 21. (Raja mobula. Lac.) Mobula orrechiuta . Tucile cora mula, ò Aquilı diarolı.

369. Ceplaleutherus maculatus. Raf. app. gen. 22. sp. 32.

Cefalotero macchiato. Tatarida dii mari, Aquilottu äi nuti.

369. Uroxis ujus. Raf. app. gen. 23. (Dasyatis ujo. Raf.car.
sp. ) Ossiuro rujo.
Lju, Buju, Vuju,
e Vughiu.

329. Apterurus fabroni. Ràf. app. gen. 24. (Raja fabroni Lac.) Atteruro di fabroui. Vacca di mari. 
371. Da syatis pastinaca. Raf. car. gen. 14. (Raja pastinnea. Linn.) Dasiata pastinaca. Vastunact, pastenaca, e forra za.

372. I Attavilla Raf. (Raja attavilla Lac ). D Dasiata attarilla. Tavila, ò Atlazida.

\section{ORDINE. I LAMPRLDINI.}

Corpo molto allungato, senza ale pettorali, ne inferiori, ne anali.

373. Petromyzon marinus. Linn. Lampreda marina.

Alampria, ò murustidela.

\section{TERZA SEZIONE. GLI ETTERITTI.}

Corpo sprovisto d'ale, e spesso anche d'occhj. Una, o due aperture branchiali da ogni lato.

\section{ORDINE. GLI ATTERIDI.}

Due occhj, un' apertura branchiale da ogni lato.

* 374. Oxrstomus hyalinus . Raf. app. gen. 25. sp. 33. Ossistomo jalino. Gujicedduz: $a$. Tar. 2. fig. 2.

* 375. Helmictis punctatus. Raf. app. gen. 26. sp. 31 . Onitto punteggiato. Pesce Tremolina, ò $\mathbf{V}$ er. miceddu Tav. 2. fig. 3.

\section{ORDINE. GLI ANOHTAIMIN1 .}

Nessun' occhio, un apertura branchiale da ogni lato. 376. Cecilia branderiana. Lac. (Murena ceca Linn.) Ceci. lia branderina. Pesce trcmolinu. IXX1. ORDINE. 1 MISSINIDI. 


\section{CATALOGO DEI NOMI SICILIANI DI ALCUN! PESCI}

Che non hò ancora visti, ne potato raguagliare metodicamente : Essi sono per lo piu di Catania, e del val di Noto, quelli segnali cost * sons di Messina, e val demme.

Pissature.

Vacauclu.

Pesce Ceccu:

Pesce Strazal.

Pesce Sbirm.

Baccuficu .

Gaduffi.

Zintalis.

Pitra

Aspereda.

Acamurgia.

Carajola.

Pesce Luvone.

Pesce gaduะঞu.

Fragulinu.

Ciolola.

Crovello.

Cossolo
Barbajolo .

Gasparcllo.

Luineru.

Surgu.

Stumbu.

Sambucu.

Scuntaru. Cata e Mess-

Zorallu.

* Birru.

* Bellicaro.

* Caccanu.

* Carcize.

* Marzoi.

* Pesce palo.

* Caragnola.

* Pampini.

* Sauru-tundu. 


\section{(31) \\ A I P E N D I"C E

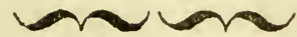

Prima Specie. Numero 9. Blennius gonocephalus. Corpo troncato, gibboso, ed angolato al disopra, senza appendici, ne barbette, corpo fosco sparso di punti, e lineette ,cerulescenti, nessuna linea laterale, ale giugulari con due raggi uguali, coda rotonda. Osseriasione. E' lungo al più di quattro pollici, ha il capo grosso, quasi rotondo, ma compresso; lc ali senza macchic, con le pettorali, e la caudale rossigne.

2. Sp. n. 14. Blennius gobioides . Capo un poco troncato, senz' appendici, ne barbette, corpo olivastro sparso di punti cc. rulescenti con delle fascie fosche sopra il dorso, c sotto il capo, nessuna linea laterale, ale giugulari con dne raggi uguali, coda rotonda. Osserv. E della grandezza del Bl. vizidus at cui rassomiglia assai, come pure al precedente; ma ha le Ale dorsali, ed anali con delle macchie jaline, e la caudale, e pettorali giallastre.

1. Genere - Gaidropsarus. Più di un raggio all' alc gitugulari, due ale dorsali, la soconda delle quali è riunita coll' s.la Gaudale, e con l' anale.

3. Sp. n. 27. Gaidropsarus mustcllaris. Quattro barbette, due alla mascella superiore, c duc all' inferioies ale griugulari eon cine raggi inugnali. Sinonimia. Mustella. Rondelet lib. 9. cap. 16. fig.

11. Gen. Strinsia. Due ale dorsali, un' ala anale riunita all' als caudale. 
4. 4p. r. 53. St insia tincte. Una barbetta sotio alla mascelu inferiore, che i pit corta della superiore, prima ala dorsale con dieci raggi .

111. Gen. Sgmphurus. Alia caudile acuta, e riunila all' alc dorsali, ed anali, occhj alla sinistra. Osscr. Si doman. no ragguaghliare in questo genere lue specie del genere Achins di Lacepede; cicè gli A. lilineatus, e A. omutus .

5. Sp. n. 44. Sympiaurus migrescens. Nerastro senza fascic, dilungato, una sola linca laterale da ogni lato.

IV. Gen. Solfa. Ale gingulari, ed ala caudale sciolte, oc: wi alla dritta .

6. Sp. n. 48. Sotca rhomboide. Coda lunutata, corpo due voite più lungo, che alto, 1 ' ala dorsale principiando avanti gुi occhi è con circa sessanta raggi, ala anale senza spina anteriurmente, linea laterale archeggiata alla base.

Sinonimiat . Mhomboide, Mondetet . liv. 11. cap. 4. fig.

Oss. Lacepede ha crechuto questo pesce una varietì della S. limanda; ma ne differisce, perchè ì più largo, e meno lungo ; dall' ali dorsale, che non principia sopra gli occhi cc.

7 Sp. n. 49. Solea cilhara. Coda rettilinca, corpo Ianciolato, tre rolte più luingo, che largo, l' ala dorsale principiando dietro gli occhj, linea laterale flessuga alla base, squame grandi, romboidali, e liscic, bocea dentata.

Sinonimia. C $i-$ tharo. Rondelet lib. 1. cap. 5. fig.

8 s.p. 11. 51. Solea arnoglossa. Coda quasi acnta, corpo bislungo biancastro, quasi tre volie più lungo, che largo, l' ala dorsale principiando sopra gli occhj, linea laterale. quasi diritta, squane piccolissime. delet lib. 11. cap. 11. fig. Sironimia, Amoglosso. Ron-

9. $s p$. 


\section{$(53)$}

9. Sp. n. j2. Soica cynoglossa. Coda rettilinea, corpor ovale, due volte più lungo, clic largo, lato dritto fusco, linea laterale curva, alia dorsale principiando sopra gli occhj, di cui l' inferiors è postcriorc al superiare . Sinzon. Cynogglosso. Rondelct. lib. 11. Can. 13. fig.

V. Gen. Scophthalmus. Mle giugulari, ed ala caudale sciolte, occhj alla sinistra.

10. Sp. n. 55. Scophtialmas diurus. Coda profondamente biforcata, corpo romboidale quasi largo quanio lungo, fosco dal lato sinistro, con delle macchic fulve, linea laterale flessuosa, ala dorsale principiando dietro gli occhj.

Sinon. Alia Passeris specics Rondelet. lib. 11. cap. 8. fig.

11 sp. n. 93. Scomber macroptithalmus. Nove raggi alla prima ala dorsale, sei piccole alette al disopra, e cinque al disotto della corla, che è indorata, o ha it seno della sua base ottuso. Osserv. Differisce dal S. Scombrus per le accennate distinzioni, oltre che ha gli occhj molto più grossi, l' iride indorata, il dorso verdastro, variegrato di linee fosche flessuose ete.

12 Sp. n. 108. Trtichurus fesciatus. Membrana branchiale con tre raggi, coda biforcata, ala anale con renti xaggi, primo raggio della prina ala dorsale quasi sciolto, e rivolto verso il capo, corpo giallo con il dorso, e delle fascie trasversaii foschiccie. Osserv. II molvo affiniti col mio T. adiciolus, ma ne difierisce dal colore, fascie, struttura della prima ala dorsale, se., oltre che è più piccolo, ha gli occhj grandi coll' iride argentina indorata, l' opercolo ̀̀ dop: jio, la linea laterale archeggiata anteriormente, le fascie so. 


\section{(54)}

5o larghe, ed al numero di quattro, o cinque; tutte le ale sono gialle, le dorsali banno la prima 8 raggi, e la seconda 30 , le pettorali 10 , e le toracine 5 .

13 sp. n. 146. Labrus mendovella. Coda biforcata, linea laterale curva posteriormente, olivastro con una fascia longitudinale rossa marginata di blù da ogni lato del capo, una macchia nera marginata di rosso sopra ogni opercolo, ed una macchietta nera da ogni lato alla base della coda. Oss.o Sarà forse una specie di Lutianus, avendo molto affinitì col $L$. olivaceus.

14 Sp. n. 147. Labrus cettii. Coda intiera, linea laterale curva posteriormente, iride rossa, macchia cerulea sopra $1^{*}$ opercolo, corpo fosco al disopra; bianchiccio al disotto, fascia longitudinale gialla sopra i fianchi. Sinon. Labrus julis . C'etti. Pcsci di Sardegna pag. 122. Osserr. Questo Labro si avvicina ben più al $L$. cynedus, che al $L$. julis, per cui lo prese Cetti, giacchè ne differisce soltanto dal colore diverso, e non dalla disposizione di tale colore.

V1. Gen. Diplodus. Una sola ala dorsale principiando vicino al capo, un appendice squamosa vicino ad ogni ala to. racina. Oss. Contiene il sparus annularis di Linneo ea Sp. Variegatus di Lacepede.

V11. Gen. Octonus . Dei raggi sciolti vicino all' ale pet. torali, una sola ala dorsale, tutto il corpo ricoperto di una corrazza ottangolare. : Oss. Differisce da questo ultimo Carattere dal genere Peristedion, che non ha che due piastre ossee sotto il corpo.

v111. Gen. Cephalepis . Corpo compressissino conico, capo 
po troneato dingonalnente, $l$ ' ano piu vicino della coda, che del capo, nessun' ala anale, un' ala dorsale lunghissima il di cui primo raggio è lenghissimo; o spatolato, ale toracine con 7. raggi tutti riuniti da una membrana, e senza nessuno scialto.

Oss. Differisce dal genere Argyctius da questo ultimo carattere, ol tre che i raggi de!la colla hanno pure una membrana: fraposta alla loro base $\&$ c.

15. Sp. n. 232. Cephalepis ocionaculatus . Argentino-ccnerino con otto macchie nere, tre ad ogni lato del dorso, una ad' ogni lato del ventre, primo raggio dell' ala dorsale bislungo alla sua estremita, coda con 6- raggi Iunghi qaasi sciolti..

Osser. Questo pesce che fù ritrovato in Messina dal Sig. Gu. glielmo Swainson naturalista studioso, che me ne comunicò un disegno, hà moltissima rassomiglianza di forma; e tutt' altro col mio.Argyctus: 4' maculatus, avendo quasi tutti l' istessi caratteri fuori di quelli accennati, le sue ale sono jaline con i raggi ro. ssici alla base, l' ale toracine sono lunghissime, ed d molto rir marchevole il prino raggio della: dorsale.

16. Sp: no. 23t. Salmo cettii. Macchiato di nero, e punteg. ziato di rosso, fosco al disopra, hianco al disotto, mascella inferiore più lunga della superiore; linea laterale quasi dritta, ala caudale lunnlata, la dorsale, ed anale con 13: raggi, di cui i tre primi sono corti, ed avvicinati, ale addominali con 9 rag. gi . Sinono Trotta Sonda. Cetti. Pesci di Sard. p. 74. Salo mo lacustris. Azuni. Descr. di Sardegna t. 11. p. 242: Oss, E lnngo un piede e mezzo circa, si ritrova in Sicilia in alcue ni fiuni del val demone, $e$ val di Noto, la seconda ala dorsa. Ie, che è adiposa, è situata al disopra dell'éstremitì dell'ala ana 


\section{(. $56 \times)$}

le, la coda tra 28 raggei, el è lunulata, ossia un poco biforcatay

17. Sp. n. 211.' Mugil ccphalus. var. 1. Cestreo. Capo piccolo, depresso, c schiacchiato: Cestreo. Rondilet. lib. 9. cap. 3. fig. Var. 2. Myxone capo piccolo; conico, maso un poco acuto. Alyxone. Rond. lib. 9. cap. 4. fig. Var. 3. Chelone. capo grosso, conico, muso ottuso. Chclone Rond. lib. 0. cap.5. fig. Oss. Differiscono tutti da questi leggicri caratteri dal vero Mugil cephalus. Céphilo Rond. lib. 9. cap. 2. fig. che hà il capo grosso, rotondio, col muso ottusissimo .

1X. Gen. Myctophim . Corpo conico, ricoperto di grande squame, capo compresso, troncato, e senza squame, bocca grande con dei picciolissimi denti alle mascelle, lingua lunga col margine ciliata, naso grande con due aperture bislunghe separate da una cresta, e marginate da un' altra; Opercoli grandissimi, aperti fino sotto alla mascella inferiore, dove si riuniscono in carena sporgente; Due ale dorsali, la seconda alliposa.

18. 5p. .. 258: Myctophum punctatum. Argentino-cerulescente, due appendici trà gli occhj, seconda ala dorsale laciniata, dei punti rotondi argentini lucenti, sparsi regolarmientelnella parte inferiore del corpo. Osserv. E lungo da tre a sei pollici, hà gli occhi grandi situati in un orbita circolare, sporgente membranoso, sono olivastri coll' iride incorata, l' ala caudale è rettilinea, fosca alla base, e con 24 raggi, la prima ala dorsale ne hà 10 , le pettorali 16 , l' addominali 10 , e l' anale 22 , 19. Sp. n. 268. Siphostoma Viridis. Verde-olivastra, con un ordine di punti bislunghi jalini da ogni lato, giallastra al disotto, 53 anelli al corpo, 35 raggi alt' ala. dorsale, bocen compressissima, ala caudale cuneiforme rotonciata. 


\section{( $5 \%)$}

X. Gen. Syngrsatlius. Un ala dorsale, ed una ala caudale, senza ale pettoraii, ne anali. Osserv. Lascio il nome di $S_{y n \text { - }}$ gnathus alla divisione di questo genere Linneano, che hà i caratteri espressi, esso contiene oltre della seguente specie, il $\mathbf{S}$. aquoreus di Linnco.

20. Sp. n. 270. Syngnathus punctatus, cilindrico, bocca ourva al disopra, un ordine di punti azzurri da ogni lato del petto, ala dorsale lunghissima .

X1. Gen. Nerophis, Un' ala dorsale, senza ale caudiali, anali, ne pettorali, corpo cilindrico. Osserv. Si compone dalla seguente specic, e del Sÿ̈gnathus ophidion di Linneo.

21. Sp. n. 271. Nerophis maculata. Ventisei raggi all'ala dorsale, senza righe longitudinali, delle macchie sparse regolar. mente. Oss. Il Syngnathus opplidion, che chiamerò Nero. phis viltata, si distingue dal suo maggior numero di rag'gi all' ala dorsale, avendone 34 , e da quattro righe longitudinali, che Jà al disopra. Il mio pesce è lungo circa un piede, ha il corpo soltile, gli occhi piccoli coll' iride olivastre, il suo colore, quello delle machie, e la loro disposione sono molto variabili; il pesce inaschio hi il ventre angoloso, ò carenato, mentre la Femina non 'vi hà nessun' ángolo, e vi porta attaccsti nella primavera due ordini di-uovi grossi, rotondi, e gialli, al numero di circa 60 ,

X11. Gen. Carapus. Nessun' ala dorsale, ne caudale, un ${ }^{2}$ ala anale, e due ale pettorali, mascella superiore più lunga dell Inferiore, coda nudi al disotto. Osserv. differisce dal vero genere Gymnotus, che hà l'ala anale lunghissima, ricuoprendo il disotto della coda, $e$ la mascella inferiore più lunga della superiore. 


\section{( 58$)$}

22. Sp. r. 292. Ostracion agonus . Corpo ellittico senza an. Goli, una spina sopra il dorso voltata verso il capo, ed un al. tra solto il ventre voltata verso la codil, che d̀ rotondia. Osserv. Hù osservato questa specic singolare nel musco del Principe di Biseari a Catanin, insieme colla seguente, é furonö ambidue pescate nel golfo chi Catania; differisce da lutti i suoi congeneri, perchì non hì il corpo angolato, hat tutte le ale ro. tonde, le due spine grosse, e forti, sporgendo orizontalmente, tulto il suo corpo è fosco senza macchie, ricoperto di una co. razza con delle divisioni romboidali, le quali hanio una linca longitudinale sporgente rel mezzo .

23. Sp, n. 294. Diodon eclinus. Corpo qunsi sferico, fosco, senza macchie, tutto ricoperto di piccole spine sottili, c non angolate coda rotonda. Osserv. Differisce dal D. Mystrix di Linneo perchè non hà macchie, che le sue spine sono più folte, più sottili, e senza veruno angolo, dà la bocca piccolis. sima, il corpo quasi inerme, le ale piccole, e la sua forma to. tale d̀ una sfera appena allungata.

X111. Gen. Sturio. Questo genere, che deve contenere lia maggiore parte delle specie del genere Acipenser, si potrà com. ponere illle specic, che hanno il muso corto, e non spatolato.

X1Y. Gen. Capriscus. Ala toracina, d inferiore con un solo raggio, diversi raggi alla prima ala dorsale. Osserviconticne la terza divisione del gencie Balistes di Lacepede."

XV. Gen. Chiopsis. Corpo cilindrico, senza squame, é con Fag, linea laterale rla ogni lato, mascelle corte, ottuse $\varepsilon_{\text {denta- }}$ te, apertura branchiale situata sotto il collo, e rotenda, l' ano pie vicino del capo che della cora, un'ala dorsale, ed un ala. 


\section{$(59)$}

aia anle riunite coll ali caudale. Osscro. Diffurisus dil genere Neticitoma par la forma dolle banihie, e quill dill. sue mascelle.

21 Sp. n. 313. Chlopsis bicolor. Ala dorsale, e linen l.. terale principiato dietro l'apertura branchiale, ctp.s, c crip), fosco al disopra, bianco al disotto, uni linea longitudinale di: punti binchi satto in linen laterule anteriornonte, inzscella sit. periore più lunge dell' inferiore, e con die pieculi appantici. Osserv. E lungo un palmo e mezzo, i due colori, cha offerisce, sono divisi senza degradazione internadia, il foseo pria. cipin sopra la mascella superiore, passa sotto gli occhi, sopra l'apertura branchiale, e sotto h lina latarale, l'ala dorsale; e la caudale sono dell' istesso colore, la prima peró tutta mar. ginata di bianco, e l'ala anale ì tutta bincr; alfias hit gli oc. chi situnti sopra l'angolo dell" apertura della boeca neri, e cora una bellissima iride, composta di tre carchi di colori diversi, it più esteriore è bianco, quallo appresso fosco, c.l il più interno d'ua rerde indorato.

XVI. Gen. Xypterus. Una sola ala dorsale, nessun'ala àa: le, ne caulale, bocci senza denti, ano pii vicino del cxpo, che della coda.

25 Sp. n. 315. Xypterus imperati. Mascelle ottuse, lin superiore più lunga, $i$ due primi ragģi dell' ala dorsale lunghis. simi, cola nudr oltusa Sironinica. Spacda marina. Imperati hist. nat. p. 6i9. \& 687. Sg.

XVII. Gen. Pterurus . Corpo allungato compresso, un'als alia cola nussun' altra, questa deccoŕrente varso il dorso, e l'ano. 23 Sp. n. 316 Ptcrurus fexuosus, mascelle ottuse, la su. periore più lunza, corpo flessuoso, jalino, coda acuta. 


\section{$(60)$}

27 Sp. r. 330. 1sirus spallenwani. Muso appantinto, denti aggrupati conici, pialti, acati, ed intieri, nessum linea la icrale, coda senza angoli laterali. Sinoniwa. Pesce Canc. Spallanani viaggrio alle due Sicille tom. 4. pag. 393. etc. Oss. Pongo questo Pesce nel genere Isurks it motivo della siz a colla lunulata secondo Spanlanzani ma se essa non sari uguale, si dorri amovwerare nel genere. Curcharias, giacchi non hà spiragli, qianli che Spallanzani cliama ejsi, sono le aperture branchiali, ed i saoi fori sono l'apritare delle narici.

23 Sp. n. 332. Cerictius caniscies - Bỉion al disopra, muso - teuso, appendici in form d'orecchie, e piegevoli, coda me. 1:0 del terzo della lunghezza totale.

XVI11. Gin. Oxynotus - Corpo triangolare, col dorso ca. renato; cinque aperture branchiali da ogni lato, due spiragli, due ale dorsali, nessun' ala anale:, coda disuguale. Osserv. differisce dal mio vero genere Squialus dalla forma del corpo, e del dorso .

XIX. Cien. Spryme. Si distingue dal mio genere Squa. lus, perchè hì il capo molto grosso, aliargato, e con gli oc. clsi situati all' estremili della parte sporgente da ogni 'lato..

XX. Gen. Torpedo. Differisce dal genere $R$ aja a motivo del suo corpo troncato anteriormente, e suoi spiragli, che so. no rotondi, staconti dagli oechi, e situatii dietro di essi.

29 Sp. v. 363. Torpclo occliaia. Cinque macchie rotonde. ngruli, disposte in forma di pentagono sopra il dorso, nere, c margginate di un cerchio biandiccio.

Sinoniuia. Torpedine $\therefore$ recondetet. lit. 12. cap. 19. fig.

93 Sp. n. 36!. Torpodo immaculata. sema macchie sopra 


\section{$(61)$}

i) dorso, ne altrove. Sinonimia. Torpedine 4. Rondelet. 31. Sp. n. 365. Torpedo punctate. Porso, ed ale sparse al cisopra di punti, e macchictte inuguali. Sironimia. Tor. pedine 3. Rondelet.

XX1. Gen. Mobula, Dorso innalzato con una sola ala, co• da nuda, capo con degli appendici al disopra.

XX11. Gen. Cephaleutherus. Capo sciolto, e diviso "dall" ale laterali, occhi, e spiragli uniti, e sitrati al lato del capo, due ale sopia la coda, nessuna alla sua estremțà .

Osserv. Questo genere è rimarcherolissimo, a motivo del carattere che offerisce il suo capo sciolto, il quale è unito all' ale laterali ossiano pettorali, in tutti gli altri generi vicini.

32 Sp. n. 368. Cephaleutherus maculatus. Fulvastro al dịn sopra con delle macchic fosche, bianchiccio al di sotto, capo appuntato, ale laterali anteriori, appuntate, e scabre anteriormente, un ordine di spine sopra la Schiena, e tre sopra la coda, che ì acuta. Ossero. Hà alcune spine disposte re. golarmente sopra il capo, e dietro gli occhi, la bocea d̀ si. tuata sotto il capo, cll in un fosso, e le aporture branchiali sono salto la parte anteriore del coxpo; cinque da ogni lato; frì le ale laterali anteriori, e posteriori vi è un appendice qua. si digitato alia panta, l' ano è più vicino del capo, che della punta della coda, questa è convessa al disopra, e piana al disotto, e lc ale, che porti, sono molto vicine alla sua estremita, approssimate frà esse, e con uua spina frì il mezzo.

XXIII. Gien. Uroxis. Differisce dal genere Daryatis, per. chè hà la coda totalmente nuda, e sprovista anche di spinc al. lungatc invece di ale. 


\section{$(0 Z)$}

XXIV. Gen. Apterurus. Nessun' ala sopra il carso, ne alla coda, capo con degli appendici .

XXVV. Gen. Oxystonus . Corpo compressissimo, e molto lungo, capo piccolo, mascelle acutissime, e dentate, coda acu-: tissima. Osserv. Queste, ed il seguente genere sono uno di quei punchi, che contengono specie sproviste totalmente d'ale; ma che nondimeno non tralasciano d' essere veri pesci.

33. Sp. n. 374. Oxystomis hyalinus, Mascell $r$ inferiore piü lunga della superiore, corpo julino, linea laterale quasi no.s visibile, tre ardini di piccolisșini punti neri sotto il ventre.

Ossero. E lungo mena d' un piede, conposto d' una sastanza gelatinosa, e traspurente, ed i suoi occhi, che sono situati sapra l' angalo della bocca, sono neri, circoadati d' ua iride argentina.

XXV1. Gen. II elmictis. Corps cilindrico, mascelle senza denti, ed ettuse, come pure la culr, ans nel in zzo lut corpo.

34. Sp. n. 375. II elmictis punctatus. Miscella superiore più lunga dell' inferiore, carps jatino-fulviccio, linez laterale conposta di un ordine di piccoli puaticcioli neri, due ordini longitudinali di simili punti sotto il corpo avauti l' ano, e tre dietro l'ano. Ossero. E lungo circe quittro pullici, e $\mathbf{b l}$ tutta l'appirenza di un verne, essald, anche flessioso, e gelatinoso, e salo lo distiagujo la sua boccl, i suoi occhi neri coll' iride argentinz, e la sur apartura bivichisle, che consiste in una piccola spaccatura traversale ad ogai lato del collo.

SUP. 


\section{( 63 )}

\section{SUP P L E M E T O :}

N. B. IIentre componevo quest' opusculo hó avuto la sort: di osṣervare alcuni altri nuovi pesci, cosicchè non sbayliai qưndo giulicui nell' introduzione, che molti averano tuttavia 'sfucsgito le mie ricrche, e vado ałmoverarli nell' istesso modo, che i pesci descritti nell' appendice.

37\%. Gadus grondoni. Gado grondone. Merluszu: di varu . Nessuna barbetta, Corpo, ed ale argentino, miscella inferiore piis lunge della supriore, coda quasi foscata, linea laterale quasi dritta, prina ala còrsale con dieci raggi .

Osseri. Questo pesce, che si ritrova pure nei mari della Sarde. gna, mi era stato conunicato da molto tempo dal Si s. Colannello Groncione di Caglieri, Ittiologo, e naturalista benemerito, ed il di cui none gli ho dato: hì alcuni tratti di somiglianza col Gudus carbonerius di Linneo, ossia il Gadus colinus di La. cepede, ma ne differisce dal colore, la minore grandezza, - ed il diverso. numero di raggi, a quasi tutte le ale, giacchè ne his 10. alla prima ala dorsale, 20. alla seconda, e 16. alla terza, oltre di 13. solamente all' ala pettorali; mentre il $G$. Garbanco rius ne hà 21. a queste, 14. alla prina dorsale, 15. alla seconda, c 20. alla terza.

378 Lutianus ruber. Luziano rosso Sapune russu, $S_{a_{\odot}}$ pune imperiali, Pisci russu, ò Pisci. beddu . Tutto rosso, pezzo posteriore dell' opercolo mutico, flessuoso, e con una macchia fosca al disopra, linea laterale curva pasteriormente, coda trifis 


\section{( ot)}

trifida con una macohietlia nera a sua base, ala dorsale con 14 raggi spinosi, e 10. articolati : Osserv. E un piccolo pesce lungo puochi pollici, che hid la bocca piccola, ma dentata, due protuberanze sopra il maso, l'ala con tre ragji spinosi, e 10. articolati, le ale toracine con un raggio spinoso, c 5 articolati, le pettorali con 12. raggi articolati, e dichotomi, c l'ala caudale con 18 raggi consimili.

XXVII. Gen. Epigonus. Corprs quasi conico, ma col dor. so un poco cirvo, e carenato, $\dot{o}$ angoloso anteriormente, due ale dorsali, becca sdentata, miso con una protuberanza al di. sopra, opercoli senza squame, ne dentature, ma angolati, e spincsí. Osserv. Appirtiene all' ordine dei Percili, e ha molta affinità col genere Ściena di Lacepede, non già quello di Linneo, ma ne differisce dalla forma del dorso, muso, bocca \&z. ed ba pure qualche somiglianza col Coltus, a ragione del suo corpo quasi conico, benchì sia però di un altro ordine.

379. Epigonus macrophthalmus . Epigono grossocchio Pesce luniu. Tutto foschiccio, mascella inferiore più lunga della superiore, opercolo bi-angolato, ed unispinoso frì gli ango. li, coda foscata, prima ala dorsale con sette raggi spinosi, $d \mathbf{~}$ cui il primo cortissimo. Osserv. I suoi occhi sono molto grossi, con l'iride argentina, la sua linea laterale è quasi drits ta, larga, e bianchiccia, ha il dorso angoloso dal capo sino alla prima ala dorsale, 8 raggi alla seconda ala dorsale, di cui il primo solo è spinoso, 10 alt" ala anale con due spinosi, edl il più anteriore cortissimo, 6 all' ale toracine, di cui uno spinoso, 15 all'ale pettorali, e 20 alla coda.

XXVIII. Gen. Gonostom «. Corpo, conico, ricoperto di gran. 


\section{$(65)$}

de squame caduche, eapo compresso, ottuso c senza squame, bocca grandissima colle mascelle angolate, c dentate, ed il palato angolato, dentato, e ciliato, narici rotonde senza creste, opercoli grandissimi, meinbranosi, ed angolati posteriormente, approssimati inferiormente in carena; una sola ala dorsale, çodạ carenata, e dentellata al disopra, ed al disotto. Osserv. Si dovrì anioverare nell' istesso ordinie, che il genere Myctophum con cui hà qualche affrnità; ma ne differisce da numenerosi ca. ratteri .

350. Gonostoma denudata. Gonostoma spogliata. Anciovo impiriciali. Coda biforcata, ala dorsale con 20 ragggi tutii articolati, ala anale con 16, dei punti argentini sotto il corpo. Ossero. 11 suo colore ̀̀ frachiccio con dei riflessi argentini, i suoi occhj sono mediocri coll' iricle indorata, e le sue ale sono senza raggi spinosi, le petrorali sono piccolissime, c con 12 raggi, l' addominali con 10 , e la coda froto 21. eltre 10 dentature spinose al dișopra, ed altrettante an

381. Torpedo maculatc. Torpilla macchiara. Tiremola tacchiata. Cinque macchic nere, rotonde, ed ugrnali, disposte sopra il dorso in forma di pentagono. Sinonimia. Torpctine 2. Rondelet.

382. Torpelo variegeata. Torpilla variçata. Tremola urdinarice Cinque macchic nere, rotonde, ed uguali, disposte sopra il dorso in forma di pentogono, con molti punti bianchicci framischiati. Ossero. Rondelezio non be mentovato quẹta Torpilla, essa è però comlane quanto le altre in Sicilia. 


\section{$(66)$}

\section{COREZZ1ON1, ED-AGGHUNiE}

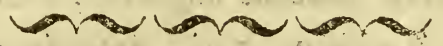

1. Il mio Centronotus binotatus $\mathrm{sp.}$ \$8. ì il giancus i. a Rondelet, che ILinaco chiamò Scomber glaucus, " Lacepe. de Carans, glaucus: dello osservazioni accurate mi hanno convinto, the non appartiene a nessuno di questi generi, ma bensi al mio aenere Iypacantho, avendo anch egli $\mathrm{i}$ raggi della prima ala torsale sciolti, -ed alcuni raggi consimili avanti dell' ala anale, dovri dunque esscre cliamato Hypacantha linotata.

2. 11 Sparus melunurus di Artedi, e Linneo, cd il mio, Sp. 15j. mi pare una specie diversa del Sparus oblada di Laeperle; benchè egli l' abbia confusi, quicllo che osservai non aveva macchie fosche ai lati, e solamente sette raggi spirnsi all' ala dorsale; ho appresso rinvenuti altri due perci consimiIi diesto in forma, ed aspetto; ma uno ha tutti $i$ caratteri Epiñ oblada di tácepede, e l' altro pare medio fro questo, ed il mio, onde lo chiamcrò $S p$. dubiüs.

3. II Salmo saurus di Linneo ossia Osmerus. saurus di Lacepede Sp. 235. dicesí da quest' ultimo autore abitante del Mediterraneo, dell' Oceano, e del mar rosso, il pesce, che lio ritrovato nel mar di Sicilia, è raguagliato is questa specie, corrisponde benissimo al Carattere specifico di Lacenede; ma è totalmente diverso dil rimanente della sua descrizione, non avendo fascie trasversali sul dorso, nc verune macchic; ma essendo quasi totalmente fulvastro con alcune righic longitudinali sopra $\mathrm{i}$ fanchi di. un bianchiccio. spor 60 , oncle mi c̀ rata l' idea, clie 


\section{$(6 \pi)$}

diverse specic siano state confuse sotte il niedesimo mentovato no: me, tutte avrebbero però l' istessa forma di capo schiacciato, carattere che contrasta con uno dei principali dei generi Salmus, ed Osmcrus, , che devono avere it capo compresso, e si rassomiglierebbero assai al mio genere Tirus, diverse sóltanto Calla mancanza della seconda ala dorsale ádiposa.

4. In rece di Onus, Ep. 30. Irggete Merlangus.

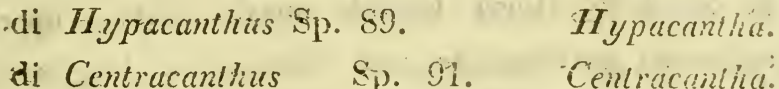

5. Mentre si stampava qnest' operetta ho osservati alcul ni altri pesci, che mi affretto di aggiungerti, cosicclie il tolale numero di quei rinventiti nel mare di Sicilia, è di S89. Specic : * 383. Phycis fuscus. Fice foschó. Pesce lupu, o lupiddu. 'Tutto foscho olivastro-, Capo appuntato, Mascella superiơre con due" barbette, e più lunga dell' inferiore, che lia una sola barbetta, ale gingulari con 4. ragsi, la prima ala dorsale con 70. raggi, ala candále rotondá.

Questa specie di Fice è distintissima da tutti suoi congeneri, è lunga puechi pollici, e benchè pare liscia è inticramente ricoperta di minutissime squame romboidali, che hanno nel mezzo un piccolo angolo sporgente longitudinale come in certe specic dell' ordine degli Adontini, ed in molti Serpenti.

* 381 Lutianus crythrophthaimus. Lutiano rosśocchio. Turduliddu. Tutto rossigno con lince longitudinali più chiare, opercoli squamosi', il posteriore mutico. e con due angoli membranosi, linea lateralc curra posteriormente, Coda rotonda gialliccia, ala dorsale con 17. raggi spinosi, e 7 articolati .

Oss. E' un piccolo pesce, che ha l' iride ros- 
sa: i raggi spinosi dell' ala dorsale sono accopiate con un filamento più lungo di essi, e una squama membranosa, ed ottusa ¿̀ fraposta tra le ale toracine, come in alcuni congeneri. 385. Sparus obląda. Lac.

Sparo oblada.

$O c_{*}$ chicta, o Ughiata.

* 386. Sparus dubius.

Sparo dubioso.

Sarpedda Coda biforcata con un cerchio foscho intorno della sua base, linea laterale quași dritti, opercolo archeggiato, argentino cerulescente con delle righe longitudinali cerulescenti . ali dorsale con 12. raggi Spinosi, e I0, articolati .

Oss. Differisce bastevolmente dallo $S p$. oblada di Lacepede dal numero dei raggi \&c. la inoltre gli occhi piceoli, e l' apparenza. della $S_{p}$. Salpa di Liuneo.

* 38\%. Sparus Spicaroides.

Spicaredda, o Mupa Spicara.
Sparo spicarella.

Coda biforcata, linea laterale dritta, opercolo otlusamente angolato, dorso rossiccio, fianchi çon alcune Iinee longitudinali giallastre, e dritte, ala dorsale eon 11. raggi spinosi, e 10 articolati. Oss. E più pic. colo del Sp. Spicara, e ha le mascelle con alcuni piccoli denți, e la superiore soltanto estensibile; una squama acuta de fraposta tra le ale toracine, come nel $S_{p}$. massiliensis di lacopede, le suc ale dorsili, pettorali, ed anali sono giallastre, ed il ventre con le ale toracine, ed anali bianchiccie, quest' ultima ha 3. raggi Spinosi, e 9. articolati.

* 383. Luçcius Vorax. Raf. ( Esox. luccius Lin.)

Iuccio vorace. Adduz $\approx a$ di. Sciume.
* Dalopleis major.
Dalofide maggriore.

Serpar sia mari.

Ala dorsale principiando quasi sopra l'aper- 
tura branchiale, dorso, e fianchi fuschi, ventre bianco, nessu. na macchia Oss. Questa specie differisce dal Dalophis Serpa per la sua lunghezza doppia al meno, ha il capo più sottile, l' ano più vicino ancora di esso, ed a qualche distanza dell' ala anale \&zc.

\section{Echclus polyrinus, Echelo polirino}

Gruncu di rina. Bigiastro al disopra, mascelle ottuse dentate, la superiore più lunga con due appendici otlusi, e die. єi narici poriformi, ale pettorali bislunghe, l' ala dorsale prinsipiando dietro, e la linea latcrale avanti di esse, ale dorsali, ef anali marginate di nero, coda quasi otlusa..

Palermo 1. Settembre $18 I 0$.

I 1 N E. 


\section{ERRATI}

pagina 6. lin. 12. aque

20

22. Caraux

. 21 : 3. TFacorus.

23 5. 187.

$\therefore 24$ 15. Mclanuus。

ส: $0,21.168$.

27 10. Apogon

$3217.28 \%$.

-48 9.864.

d. $0 \quad 15.336$.
CORREZIONI

leg. acque .

Caranx

Trachurus

137

Melanurus

158.

Mullus Apogon

237.

364.

360. 
cy. 2

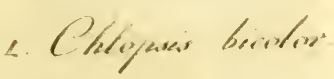

Cilcouse beschoor

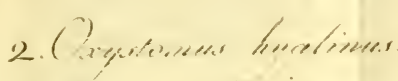
(Depreomer jépertin

3.C Helueceris fuencrenters.

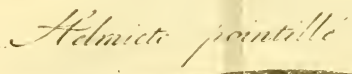

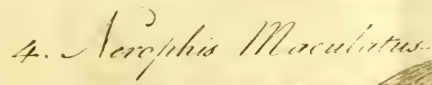

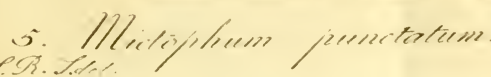

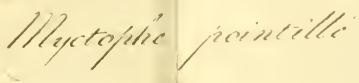

stim 



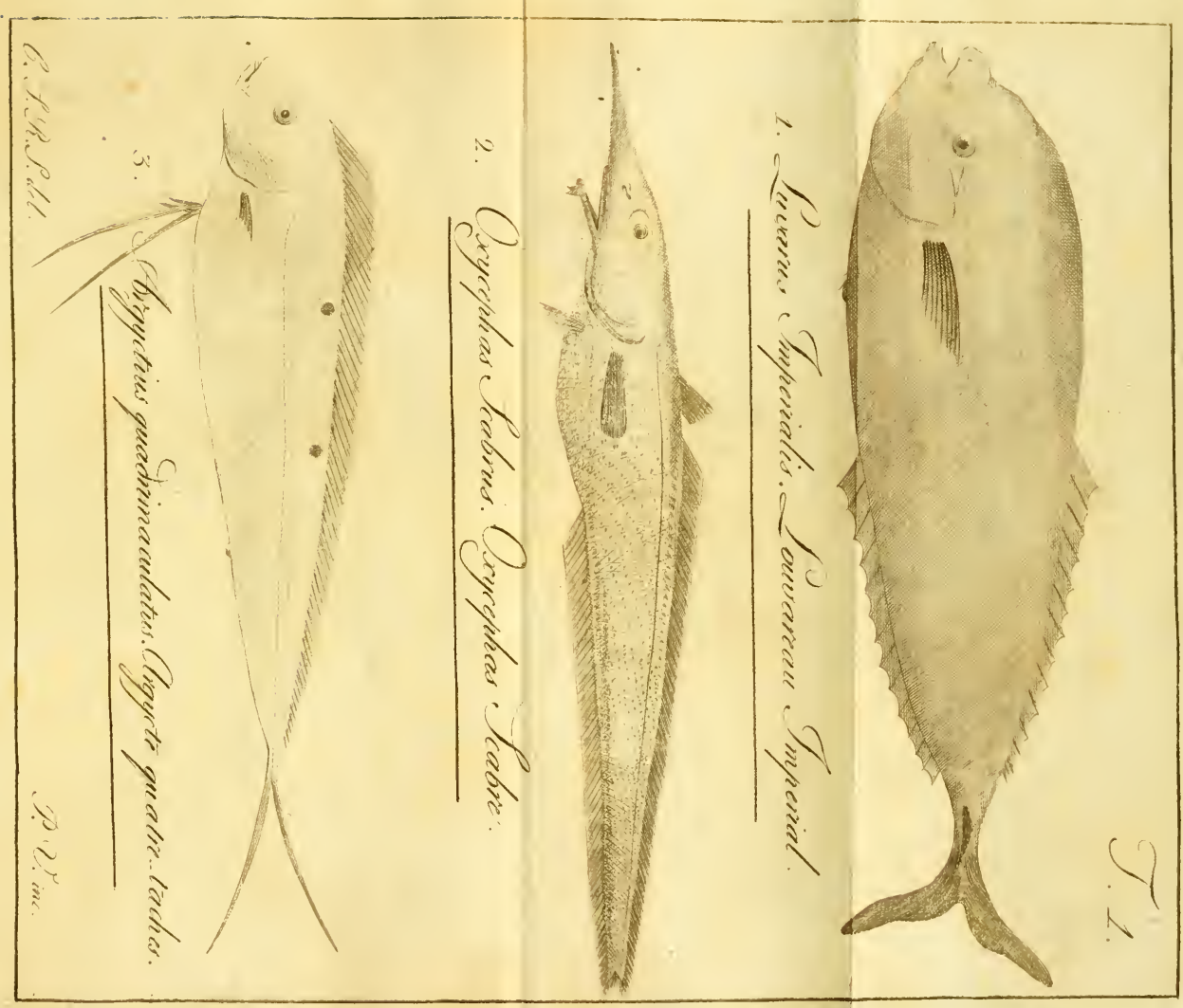









